\title{
Higher-spin gravity and torsion on quantized space-time in matrix models
}

\author{
Harold C. Steinacker \\ Faculty of Physics, University of Vienna, \\ Boltzmanngasse 5, A-1090 Vienna, Austria \\ E-mail: harold.steinacker@univie.ac.at
}

AbStRact: A geometric formalism is developed which allows to describe the non-linear regime of higher-spin gravity emerging on a cosmological quantum space-time in the IKKT matrix model. The vacuum solutions are Ricci-flat up to an effective vacuum energymomentum tensor quadratic in the torsion, which arises from a Weitzenböck-type higher spin connection. Torsion is expected to be significant only at cosmic scales and around very massive objects, and could behave like dark matter. A non-linear equation for the torsion tensor is found, which encodes the Yang-Mills equations of the matrix model. The metric and torsion transform covariantly under a higher-spin generalization of volume-preserving diffeomorphisms, which arises from the gauge invariance of the matrix model.

KeYwords: Higher Spin Gravity, M(atrix) Theories, Models of Quantum Gravity, NonCommutative Geometry

ARXIV EPRINT: 2002.02742 


\section{Contents}

1 Introduction 1

2 Matrix model and cosmological spacetime solution 4

2.1 Bundle structure and higher-spin fields as push-forwards $\quad 7$

2.2 Scales and orders of magnitude $\quad 9$

$\begin{array}{lll}2.3 & \text { Poisson bracket and reduction to } \mathcal{M} & 11\end{array}$

$3 \quad$ Higher-spin gauge invariance and Lie derivatives $\quad 12$

$\begin{array}{ll}3.1 \text { Gauge transformation of scalar fields } & 12\end{array}$

$\begin{array}{lll}3.2 & \text { Gauge transformation of vector fields } & 13\end{array}$

4 Kinetic action, frame and effective metric on $\mathcal{M} \quad 14$

5 Geometric description of the non-linear regime $\quad 17$

$\begin{array}{lll}5.1 & \text { Weitzenböck connection and torsion } & 17\end{array}$

$\begin{array}{lll}5.2 & \text { Some useful identities } & 21\end{array}$

5.3 Equation of motion for the torsion 22

$\begin{array}{ll}5.4 \text { Bianchi identity } 24 & 24\end{array}$

5.5 Vacuum equation for the Ricci tensor 24

5.6 Discussion and further considerations 26

6 Conclusion and outlook 28

$\begin{array}{ll}\text { A Technical supplements } & \mathbf{3 0}\end{array}$

$\begin{array}{lll}\text { A.1 Volume-preserving diffeomorphisms } & 30\end{array}$

A.2 Calculations for the Poisson bracket 30

A.3 Proof of Lemma 5.1 32

A.4 Weitzenböck connection for the cosmic background 33

A.5 Alternative derivation of the Ricci tensor in vaccum 35

$\begin{array}{lll}\text { A.6 Contractions of the (con)torsion } & 37\end{array}$

\section{Introduction}

Our present understanding of gravity in terms of general relativity (GR) is incomplete for several reasons. One problem is that GR is not renormalizable [1], and hence does not define a unique quantum theory. This is reflected in notorious difficulties trying to quantize various formulations of GR. Broader approaches towards a quantum theory of gravity include notably string theory, which leads to gravity in 10 dimensions. However, ad-hoc reductions to $3+1$ dimensions lead to a lack of predictivity known as the landscape problem. 
Since gravity is tied to the structure of space-time, a natural strategy is to develop a suitable "quantum" framework for space-time, based on generalized or noncommutative notions of geometry. However, there is little reason to expect that straightforward attempts to mimic GR in such a framework would overcome these issues. Moreover, pathological long-distance effects arise generically on non-commutative spaces due to virtual stringlike non-local modes, known as UV/IR mixing [2-4]. They cancel only in the maximally supersymmetric IKKT matrix model [5], which should thus have the best chance to describe physics, leading to an unexpected link with string theory.

However, it is not obvious how to obtain gravity from Yang-Mills-type matrix models such as the IKKT model. There are intriguing hints such as non-local gauge transformations and Ricci-flat propagating metric fluctuations [6-8], but the presence of an antisymmetric tensor $\theta^{\mu \nu}$ in space-time leads to a dangerous breaking of Lorentz invariance. This problem can be overcome by considering a higher-spin generalization, where $\theta^{\mu \nu}$ is replaced by a twisted bundle of such tensors over space-time. This is realized in a simple solution of the IKKT model with a mass term interpreted as cosmological FLRW space-time $\mathcal{M}$, based on the doubleton representations of $\mathfrak{s o}(4,2)[9,10]$, cf. [11]. It leads to a higher spin gauge theory which is invariant under a higher spin generalization of volume-preserving diffeomorphisms, ghost-free at the linearized level, and includes spin 2 gravitons. A linearized Schwarzschild-like solution was also found [12]. The theory has intriguing structural similarities with Vasilievs higher-spin gravity $[13,14]$, but also crucial differences: ${ }^{1}$ it is defined through an action, both IR and UV scale parameters are present, and there are 5 propagating metric modes which could be interpreted as "would-be massive" gravitons.

In the present paper, we study that higher-spin theory at the non-linear level. This is not straightforward because the model is of Yang-Mills type, there is no Einstein-Hilbert action, and everything is based on Poisson brackets (or commutators). The gauge symmetry corresponds to generalized diffeomorphisms rather than local Lorentz transformations, ${ }^{2}$ hence it is not some reformulation of GR in the spirit of MacDowell-Mansouri [23]. A more appropriate approach can be found in a paper by Langmann and Szabo (LS) [24], who pointed out that a dimensional reduction of a gauge theory in 8-dimensional phase space with suitable constraints can be interpreted in terms of 4-dimensional teleparallel gravity through torsion. Although their setup does not provide a complete theory, a similar strategy provides a geometric understanding of the present model. See also $[25,26]$ for related work.

The first message of the present paper is indeed that torsion, rather than curvature, is the key to a geometric understanding of the matrix model. The reason is quite simple: the matrices $Z^{a}$, which are the fundamental degrees of freedom of the model (2.1), naturally define a frame $E^{a}=\left[Z^{a},.\right]$, which defines the effective metric governing all propagating modes. Given this intrinsic frame, it is natural to consider the associated Weitzenböck connection $\nabla$, which is defined by $\nabla E^{a}=0$. This connection has no curvature but torsion. The crucial

\footnotetext{
${ }^{1}$ It may also be interesting to compare it with chiral higher spin gravity [15, 16], where an action can be written in light-cone gauge. An action formulation is also possible for conformal higher spin gravity $[17,18]$, notably in 3 dimensions [19-21].

${ }^{2} \mathrm{~A}$ somewhat related proposal was put forward in [22] where the matrices are interpreted as covariant derivatives, which formally leads to the Einstein equations. However this is not quite borne out here.
} 
observation is that this torsion naturally encodes the field strength $\left[Z^{a}, Z^{b}\right]$ of the model, which underlies the noncommutative gauge theory. We will obtain a non-linear geometric equation for the torsion, which fully captures the underlying Yang-Mills equations of motion of the matrix model. This provides a useful description of the resulting higher-spin gravity on $\mathcal{M}$. The Ricci tensor of the effective metric can then be computed using the equation of motion for the torsion. It turns out that torsion leads to a specific and very interesting modification of the Einstein equations in vacuum here, in contrast to LS or teleparallel gravity.

At a more technical level, the present model can be viewed as a reformulation (rather than a reduction) of a 6-dimensional gauge theory to 4-dimensional higher-spin gravity via torsion. The 6-dimensional gauge theory lives on fuzzy $\mathbb{C} P^{1,2}$, which is a quantized $S^{2}$ bundle over a $3+1$-dimensional FLRW space-time $\mathcal{M}$. This is the geometrical description of the underlying background solution as a coadjoint orbit of $\mathrm{SO}(4,2)$. The local stabilizer group of a point on $\mathcal{M}$ acts non-trivially on the local $S^{2}$ fiber, so that the would-be Kaluza Klein modes become higher spin modes, leading to a higher spin theory on $\mathcal{M}[9,27]$. The concept of torsion allows to fully describe this gauge theory in terms of higher-spin gravity. The gauge transformations of the underlying matrix model lead to covariant transformation laws for the metric and torsion, in terms of generalized (higher-spin) Lie derivatives. Although the underlying gauge invariance is exact, its reformulation in terms of 4-dimensional geometry is valid only in an asymptotic regime. Thus gravity and general covariance are understood as emergent phenomena.

The main result of this paper is a closed system of non-linear equations for the metric and the torsion in vacuum. Torsion is governed by a Yang-Mills-like equation (5.47) supplemented by a Bianchi identity (5.54), and the Einstein equation is obtained with an effective energy-momentum tensor due to torsion (5.72). These are equations for higher-spin valued fields. ${ }^{3}$ The cosmological background provides an exact solution with torsion but without higher spin components, corresponding to $\omega=-\frac{1}{3}$ in vacuum. Torsion generally leads to deviations from Ricci-flatness, however this effect is typically small in a weak gravity regime, as the energy-momentum tensor for torsion is quadratic. Nevertheless, a rough qualitative estimate suggests that torsion might lead to an apparent "dark matter halo" around very massive objects.

Most importantly, the present model has a non-perturbative definition as a Yang-Mills type matrix model, which should make sense at the quantum level. There is indeed intriguing evidence from numerical simulations $[28,29]$ that an expanding $3+1$-dimensional spacetime structure arises at the non-perturbative level. It should therefore be possible in principle to test, justify and possibly improve the analytical studies with numerical simulations.

This paper considers only the vacuum sector of the theory, and the main open issue is how matter acts as a source of torsion and curvature. It is clear that the propagation of matter is governed by the effective metric, and the manifest covariance strongly suggests that matter will lead to the usual source term for the Einstein tensor. However extra derivative terms should be expected, and quantum effects may be important here. This needs to be clarified in future work.

\footnotetext{
${ }^{3}$ For the higher spin contributions, the equations are obtained only in an asymptotic regime, for wavelengths much shorter than the cosmic scale.
} 
The outline of the paper is as follows. We first recall the semi-classical description of the space-time and the underlying bundle in terms of Poisson manifolds. A mathematical formalism is developed in section 2.1, where the bundle structure is translated into higherspin valued fields on space-time. Higher-spin valued Lie derivatives are introduced in section 3, which are the key to covariance under higher-spin valued diffeomorphisms. The kinematical setup of the matrix model is translated into this language in section 4 , which allows to derive the equation of motion for torsion and the Ricci tensor in sections 5.3 and 5.5. To validate these results, a more pedestrian derivation of the latter is given in section A.5. The possible role of torsion as dark matter candidate is briefly discussed. Some technical considerations are delegated to the appendix, including the computation of the vacuum geometry in section A.4.

\section{Matrix model and cosmological spacetime solution}

Our starting point is the IKKT or IIB matrix model extended by a mass term,

$$
S[Z, \Psi]=\operatorname{Tr}\left(\left[Z^{a}, Z^{b}\right]\left[Z_{a}, Z_{b}\right]+2 m^{2} Z_{a} Z^{a}+\bar{\Psi} \Gamma_{a}\left[Z^{a}, \Psi\right]\right)
$$

where $Z^{a} \in \operatorname{End}(\mathcal{H}), a=0, \ldots, 9$ are hermitian matrices acting on a Hilbert space $\mathcal{H}$, and $\Psi$ are Majorana-Weyl spinors whose entries are (Grassmann-valued) matrices. Indices are contracted with the $\mathrm{SO}(9,1)$-invariant tensor $\eta^{a b}$. This model has a manifest $\mathrm{SO}(9,1)$ symmetry, and it is invariant under gauge transformations

$$
Z^{a} \rightarrow U Z^{a} U^{-1}
$$

by unitary matrices $U \in U(\mathcal{H})$. The model is maximally supersymmetric for $m^{2}=0$ which is important for its quantization, but we will focus on the bosonic sector here. The only mathematical structures in the matrix model are matrices and commutators, which reduce to functions and Poisson brackets in the semi-classical limit. We must hence learn how to efficiently work with these, and to cast the system into a recognizable geometric form. This depends very much on the background under consideration. Dropping the fermions, the model (2.1) leads to the following equations of motion

$$
\left[Z_{b},\left[Z^{b}, Z^{a}\right]\right]=m^{2} Z^{a} .
$$

Doubletons and quantized algebra of functions. The basic solution under consideration here is based on special representations of $\mathfrak{s o}(4,2)$. Let $M^{a b}$ be $\mathfrak{s o}(4,2)$ generators, which satisfy

$$
\left[M_{a b}, M_{c d}\right]=i\left(\eta_{a c} M_{b d}-\eta_{a d} M_{b c}-\eta_{b c} M_{a d}+\eta_{b d} M_{a c}\right)
$$

with indices $a, b=0, \ldots, 5$. Now consider the doubleton representations $\mathcal{H}_{n}$ for $n \in \mathbb{N}$, which are minimal unitary highest-weight irreps which remain irreducible under $\mathrm{SO}(4,1)$. Then the matrices

$$
\begin{aligned}
T^{\mu} & =\frac{1}{R} M^{\mu 4}, & \mu & =0, \ldots, 3 \\
X^{\mu} & =r M^{\mu 5}, & X^{4} & =r M^{45}
\end{aligned}
$$


satisfy the commutation relations

$$
\begin{aligned}
{\left[X^{\mu}, X^{\nu}\right] } & =-i r^{2} M^{\mu \nu}=: i \Theta^{\mu \nu} \\
{\left[T^{\mu}, X^{\nu}\right] } & =\frac{i}{R} \eta^{\mu \nu} X^{4} \\
{\left[T^{\mu}, T^{\nu}\right] } & =-\frac{i}{r^{2} R^{2}} \Theta^{\mu \nu}
\end{aligned}
$$

It turns out that the operator algebra $\operatorname{End}\left(\mathcal{H}_{n}\right)$ can be viewed as quantized algebra of functions on a $S^{2}$ bundle over some $3+1$-dimensional space-time $\mathcal{M}$. To see this, consider the semi-classical limit $n \rightarrow \infty$ indicated by $\sim$, where $\operatorname{End}\left(\mathcal{H}_{n}\right)$ becomes a commutative algebra $\mathcal{C}$ of functions generated by $x^{\mu} \sim X^{\mu}$ and $t^{\mu} \sim T^{\mu}$, with Poisson brackets $\{.,.\} \sim-i[.,$. arising from the commutation relations. For the doubleton representations under consideration, these generators satisfy additional relations, which in the semi-classical limit reduce to

$$
\begin{array}{rlr}
x_{\mu} x^{\mu} & =-R^{2}-x_{4}^{2}=-R^{2} \cosh ^{2}(\eta), & R \sim \frac{r}{2} n \\
t_{\mu} t^{\mu} & =r^{-2} \cosh ^{2}(\eta), \\
t_{\mu} x^{\mu} & =0 \\
t_{\mu} \theta^{\mu \alpha} & =-\sinh (\eta) x^{\alpha} \\
x_{\mu} \theta^{\mu \alpha} & =-r^{2} R^{2} \sinh (\eta) t^{\alpha} \\
\eta_{\mu \nu} \theta^{\mu \alpha} \theta^{\nu \beta} & =R^{2} r^{2} \eta^{\alpha \beta}-R^{2} r^{4} t^{\alpha} t^{\beta}+r^{2} x^{\alpha} x^{\beta}
\end{array}
$$

where $\mu, \alpha=0, \ldots, 3$. One can also show that $\theta^{\mu \nu}$ can be expressed as [9]

$$
\theta^{\mu \nu}=\frac{r^{2}}{\cosh ^{2}(\eta)}\left(\sinh (\eta)\left(x^{\mu} t^{\nu}-x^{\nu} t^{\mu}\right)+\epsilon^{\mu \nu \alpha \beta} x_{\alpha} t_{\beta}\right) .
$$

Thus $X^{\mu}$ can be interpreted as quantized functions

$$
X^{\mu} \sim x^{\mu}: \quad \mathcal{M} \hookrightarrow \mathbb{R}^{3,1}, \quad \mu=0, \ldots, 3,
$$

onto the region $-x_{\mu} x^{\mu} \geq R^{2}$. $\mathcal{M}$ turns out to be a two-sheeted cosmological FLRW space-time with manifest $\mathrm{SO}(3,1)$ symmetry. Here $\eta$ is a global time coordinate defined by

$$
R \sinh (\eta)= \pm \sqrt{-x_{\mu} x^{\mu}-R^{2}}=x^{4},
$$

which is related to the scale parameter of the universe [12]

$$
a(t)^{2}=R^{2} \cosh ^{2}(\eta) \sinh (\eta) .
$$

The sign of $\eta$ separates the two sheets of $\mathcal{M}$, with a big bounce at $\eta=0$. Similarly, the $t^{\mu}$ are extra generators which describe the internal $S^{2}$ fiber over every point on $\mathcal{M}$, see figure 1. This $S^{2}$ is space-like due to $(2.7 \mathrm{c})$ with radius $r^{-2} \cosh ^{2}(\eta)$. Together, $x^{\mu}$ and $t^{\mu}$ generate the algebra $\mathcal{C} \cong \mathcal{C}^{\infty}\left(\mathbb{C} P^{1,2}\right)$ of functions on a 6 -dimensional bundle, which turns out to be $\mathbb{C} P^{1,2}$. This is an $\mathrm{SO}(4,1)$ - equivariant bundle over $H^{4}$, which projects onto $\mathcal{M}$. As explained in $[9,30]$ there is an equivariant quantization map

$$
\mathcal{Q}: \quad \mathcal{C}=\mathcal{C}^{\infty}\left(\mathbb{C} P^{1,2}\right) \rightarrow \operatorname{End}\left(\mathcal{H}_{n}\right)
$$




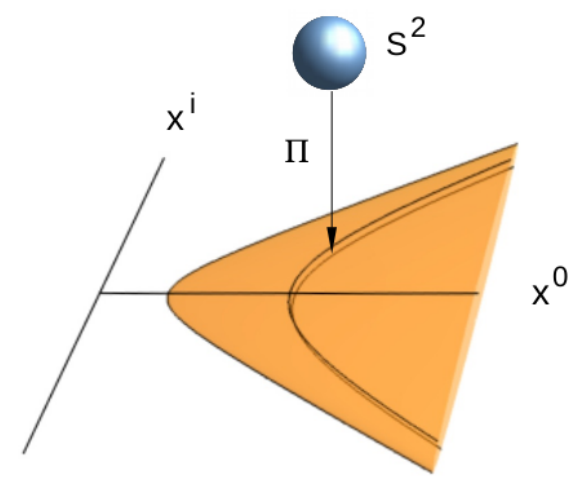

Figure 1. $S^{2}$ bundle over $\mathcal{M}$ with bundle projection $\Pi$.

which allows to identify operators with functions up to some cutoff. This paper is devoted to the semi-classical limit, replacing the r.h.s. with the lhs, and commutators by Poisson brackets. In particular, the relation (2.6b) implies that the derivations

$$
-i\left[T^{\mu}, .\right] \sim\left\{t^{\mu}, .\right\}=\sinh (\eta) \partial_{\mu}
$$

act as momentum generators on $\mathcal{M}$, leading to the useful relation

$$
\partial_{\mu} \phi=\frac{1}{\sinh (\eta)}\left\{t_{\mu}, \phi\right\}
$$

for $\phi=\phi(x)$.

Higher spin sectors and Poisson brackets. By expanding the algebra $\mathcal{C}$ into polynomials of minimal degree $s$ in $t^{\mu}$, we obtain a decomposition

$$
\mathcal{C}=\mathcal{C}^{\infty}\left(\mathbb{C} P^{1,2}\right)=\bigoplus_{s=0}^{\infty} \mathcal{C}^{s}
$$

into spin $s$ sectors $\mathcal{C}^{s}$. These sectors can also be defined in terms of a $\mathfrak{s o}(4,2)$ Casimir [9]. Here $\mathcal{C}^{0}$ are functions of $x$, and $\mathcal{C}^{s} \ni \phi_{\underline{\alpha}}(x) t^{\underline{\alpha}}$ where $t^{\underline{\alpha}} \equiv t^{\alpha_{1} \ldots \alpha_{s}}$. The projection of $\phi \in \mathcal{C}$ to $\mathcal{C}^{s}$ will be denoted by $[\phi]_{s}$ or $\phi^{(s)}$. The constraints $(2.7)$ reveal that the multiplication respects this grading as follows:

$$
\mathcal{C}^{s} \cdot \mathcal{C}^{s^{\prime}} \in \mathcal{C}^{s+s^{\prime}} \oplus \mathcal{C}^{s+s^{\prime}-2} \oplus \ldots \oplus \mathcal{C}^{\left|s-s^{\prime}\right|} .
$$

In particular, each $\mathcal{C}^{s}$ is a $\mathcal{C}^{0}$ module, so that $\mathcal{C}$ can be viewed as space of sections of some vector bundle over $\mathcal{M}$. This is a module of higher-spin fields, and the bundle structure is encoded in the sub-algebra $\mathcal{C}^{0} \subset \mathcal{C}$. The Poisson structure respects this as follows:

$$
\begin{aligned}
\left\{t, \mathcal{C}^{s}\right\} & \in \mathcal{C}^{s} \\
\left\{x, \mathcal{C}^{s}\right\} & \in \mathcal{C}^{s+1} \oplus \mathcal{C}^{s-1}
\end{aligned}
$$


and the general structure follows from the derivation property:

$$
\left\{\phi_{\alpha}(x) t^{\alpha}, \mathcal{C}^{s}\right\}=\phi_{\alpha}(x)\left\{t^{\alpha}, \mathcal{C}^{s}\right\}+t^{\alpha}\left\{\phi_{\alpha}(x), \mathcal{C}^{s}\right\} \quad \in \mathcal{C}^{s+2} \oplus \mathcal{C}^{s} \oplus \mathcal{C}^{s-2} .
$$

This is a rather complicated structure, but we will argue in the following that typically the terms involving space-time derivatives $\partial$ are dominant, except possibly in the extreme IR.

Background solution and linearized fluctuations. The above geometry provides the geometric interpretation of the following solution of (2.3) [9]

$$
\bar{Z}^{\mu}=T^{\mu}=\frac{1}{R} M^{\mu 4}, \quad \mu=0, \ldots, 3 \quad \text { for } \quad R^{-2}=\frac{1}{3} m^{2}
$$

with all remaining matrices $\bar{Z}^{a}, a=4, \ldots, 9$ set to zero. This is the cosmic background under consideration. We will also consider more general solutions $Z^{\mu} \in \operatorname{End}\left(\mathcal{H}_{n}\right)$ of $(2.3)$, which can be viewed as deformations thereof. Any such background defines a matrix d'Alembertian

$$
\square:=\left[Z^{\mu},\left[Z_{\mu}, .\right]\right] \sim-\left\{Z^{\mu},\left\{Z_{\mu}, .\right\}\right\}
$$

which acts on $\phi \in \operatorname{End}\left(\mathcal{H}_{n}\right) \sim \mathcal{C}^{\infty}\left(\mathbb{C} P^{1,2}\right)$, and will play a central role in the following. In the semi-classical limit, we can consider these $Z^{\mu}$ as elements of the Poisson algebra $\mathcal{C}$ using the correspondence (2.12), i.e. as functions on the cosmic background.

On any such background $Z^{\mu}$, the matrix model defines an action for the fluctuations

$$
Z^{\mu} \rightarrow Z^{\mu}+\mathcal{A}^{\mu}, \quad \mathcal{A}^{\mu} \in \mathcal{C} .
$$

We briefly recall the main results for these fluctuation modes $\mathcal{A}$, which transform under a higher-spin-type gauge invariance inherited from (2.2). The spectrum of square-integrable on-and off-shell modes $\mathcal{A}$ on $\bar{Z}^{\mu}$ was fully classified in [10]. It turns out that off-shell, there are 4 towers of higher spin modes for any spin $s>0$, and 2 towers for $s=0$. Among these, there are two towers of physical (=gauge-fixed on-shell) higher spin modes for any spin $s \geq 1$. These towers are truncated with maximal $\operatorname{spin}^{4} n$. In the semi-classical limit $n \rightarrow \infty$, the truncation disappears, and all physical modes are shown to have positivedefinite inner product.

In the remainder of this paper, we will develop a geometric description of the vacuum solutions in the non-linear regime, avoiding the split into background and fluctuations. The detailed relation with the above modes should be clarified in future work.

\subsection{Bundle structure and higher-spin fields as push-forwards}

The crucial geometrical observation is as follows. The bundle projection $\Pi: \mathbb{C} P^{1,2} \rightarrow \mathcal{M}$ (see figure 1) allows to map vectors from $\mathbb{C} P^{1,2}$ to $\mathcal{M}$ via the push-forward, but it does not

\footnotetext{
${ }^{4}$ There is a subtlety due to the fact that only the space-like local rotations are manifest on the FLRW background but boosts are not. With "spin $s$ modes" we refer to the 4-dimensional spin from the underlying $H^{4}$ point of view, which contain $2 s+1$ degrees of freedom corresponding to massive modes. However the mass is essentially zero, so that they decompose further under the FLRW isometry group $\operatorname{SO}(3,1)$ into different massless (transverse traceless) modes. For example, the 5 dof of a spin 2 mode decomposes into 2 dof of a massless graviton, 2 dof of a massless vector field and a scalar. For further details we refer to [9, 10].
} 
map vector fields on $\mathbb{C} P^{1,2}$ to vector fields on $\mathcal{M}$ because $\Pi$ is not injective. However, one can make sense of this as push-forward of vector fields on $\mathbb{C} P^{1,2}$ to $\mathfrak{h} \mathfrak{s}$-valued vector fields on $\mathcal{M}$,

$$
\Pi_{*}: \quad \Gamma \mathbb{C} P^{1,2} \rightarrow \Gamma \mathcal{M} \otimes \mathfrak{h} \mathfrak{s}
$$

and similarly for tensor products of vector fields. Here $\mathfrak{h} \mathfrak{s}$ is the space of functions on the fiber $S^{2}$, which is spanned by polynomials in the $t^{\mu}$. The algebra $\mathcal{C}$ of functions on $\mathbb{C} P^{1,2}$ is viewed as algebra of $\mathfrak{h} \mathfrak{s}$-valued functions on $\mathcal{M}$. This is the crucial concept which will allow to translate the gauge theory on $\mathbb{C} P^{1,2}$ as a higher-spin gauge theory on $\mathcal{M}$, which can be viewed as generalized gravity theory. ${ }^{5}$ Note that the map $(2.22)$ respects ${ }^{6} \mathrm{SO}(3,1)$, which implies that the modes in $\mathfrak{h} \mathfrak{s}$ translate into higher-spin modes on $\mathcal{M}$. However in contrast to Vasiliev's higher spin gravity, the algebra generated by the $t^{\mu}$ is commutative (in the semi-classical limit under consideration), and their nonabelian structure is encoded in their Poisson brackets $\left\{t^{\mu}, t^{\nu}\right\}=-\frac{1}{r^{2} R^{2}} \theta^{\mu \nu}$ (2.6c). As a vector space, the constraints including (2.7) encode the degrees of freedom ${ }^{7}$ a $\mathfrak{h} \mathfrak{s}$-algebra in 4 dimensions at each point on $\mathcal{M}$; for more details see e.g. section 4 in [31].

There are hence 2 different points of view of the present structure. One can take the 6-dimensional picture of $\mathbb{C} P^{1,2}$ as a 6-dimensional manifold, which is a bundle over $\mathcal{M}$. However from the physical point of view, it is better to view this structure in terms of $\mathfrak{h} \mathfrak{s}$-valued functions and tensors on $\mathcal{M}$. We will use both points of view in this paper as appropriate, but emphasize the more physics-oriented 3+1-dimensional picture.

Poisson structure and Hamiltonian vector fields. As usual, the Poisson structure on $\mathcal{C}$ allows to associate to any $\Lambda \in \mathcal{C}$ a vector field on $\mathbb{C} P^{1,2}$, via

$$
\{\Lambda, \phi\} \equiv \mathcal{L}_{\xi} \phi, \quad \Lambda=\Lambda^{*} \in \mathcal{C} .
$$

This is the Lie derivative of $\phi \in \mathcal{C}^{\infty}\left(\mathbb{C} P^{1,2}\right)$ along the "Hamiltonian" vector field $\xi=$ $\{\Lambda,$.$\} , which defines a (one-parameter family of) diffeomorphism on \mathbb{C} P^{1,2}$, more precisely a symplectomorphism. Restricted to $\mathcal{C}^{0} \subset \mathcal{C}$, this takes the familiar form

$$
\delta_{\Lambda} \phi^{(0)}=\mathcal{L}_{\xi} \phi^{(0)}=\xi^{\mu} \partial_{\mu} \phi^{(0)}
$$

where

$$
\xi=\xi^{\mu} \partial_{\mu}, \quad \xi^{\mu}=\left\{\Lambda, x^{\mu}\right\}
$$

This is nothing but the push-forward $\Pi_{*} \xi$ as in (2.22), and we will use the same symbol $\xi$ for both pictures. Clearly the components $\xi^{\mu}$ have in general components in different $\mathcal{C}^{s}$ sectors, which means that $\xi$ is viewed as $\mathfrak{h} \mathfrak{s}$-valued vector field on $\mathcal{M}$.

Similarly, we can also consider the push-forward of the Poisson structure or bivector field $\{.,$.$\} on \mathbb{C} P^{1,2}$ as $\mathfrak{h} \mathfrak{s - v a l u e d}$ Poisson structure on $\mathcal{M}$, denoted as

$$
\{\phi, \psi\}_{\mathcal{M}}=\theta^{\mu \nu} \partial_{\mu} \phi \partial_{\nu} \psi
$$

\footnotetext{
${ }^{5}$ This is the crucial step beyond the work [24].

${ }^{6}$ This is the structure of an equivariant bundle, which is in contrast to standard Kaluza-Klein reduction, where the stabilizer group of a point on the base does not act on the fiber.

${ }^{7}$ The relation with e.g. $\mathfrak{h} \mathfrak{s}(s o(3,2))$ is more visible using the Euclidean point of view on fuzzy $H_{n}^{4}$. The present Minkowski version coincides as a vector space but has modified covariance properties.
} 
Note that $\theta^{\mu \nu}=\left\{x^{\mu}, x^{\nu}\right\}$ takes indeed values in $\mathcal{C}^{1}$, and any coordinates on $\mathcal{M}$ can be used here. This respects the Jacobi identity as long as it acts on $\mathcal{C}^{0}$, but not in general since the push-forward kills vertical vector fields. The push-forward basically means that $\mathfrak{h s}$ (or the fiber coordinates) is considered as commutative. We will see that replacing $\{.,$.$\} by \{., .\}_{\mathcal{M}}$ is a good approximation for functions whose wavelengths are much shorter than cosmic scales and for low spin, since then the Jacobi identity holds to a very good approximation. This will be justified in the next sections. Then $\xi=\{\Lambda, .\}_{\mathcal{M}}$ defines a $\mathfrak{h} \mathfrak{s}-$ valued Hamiltonian vector field on $\mathcal{M}$, and we can drop the subscript $\mathcal{M}$ in the appropriate regime. No information is lost in this step, due to the following Lemma:

Lemma 2.1. Hamiltonian vector fields $\{\Lambda,$.$\} on \mathcal{C}$ are uniquely determined by their action on $\mathcal{C}^{0}$, i.e. if $\left\{\Lambda, x^{\mu}\right\}=0 \forall \mu$ (in some open neighborhood in $\mathcal{M}$ ), then $\{\Lambda,.\} \equiv 0$ (in the open neighborhood in $\mathcal{M}$ ).

Proof. This follows from the Jacobi identity, since

$$
\left\{\Lambda, \theta^{\mu \nu}\right\}=\left\{\Lambda,\left\{x^{\mu}, x^{\nu}\right\}\right\}=-\left\{x^{\mu},\left\{x^{\nu}, \Lambda\right\}\right\}-\left\{x^{\nu},\left\{\Lambda, x^{\mu}\right\}\right\}=0
$$

and $\theta^{\mu \nu}$ together with $x^{\mu}$ generate the full algebra $\mathcal{C}$.

The following two sections provide a more detailed justification for a certain approximation, which is used later to obtain the reduced 3+1-dimensional equations. Although this is very important, one may skip these sections at first reading and jump to section 3 , where the higher-spin gauge invariance is discussed.

\subsection{Scales and orders of magnitude}

Using $\mathrm{SO}(3,1)$ invariance, we can restrict ourselves to the "reference point" $p=\left(x^{0}, 0,0,0\right)$ on $\mathcal{M}$. Then $(2.8)$ reduces to

$$
\begin{aligned}
& \theta^{0 i} \stackrel{p}{=} \frac{r^{2}}{\cosh ^{2}(\eta)} \sinh (\eta) x^{0} t^{i} \sim r^{2} R t^{i} \\
& \theta^{i j} \stackrel{p}{=} \frac{r^{2}}{\cosh ^{2}(\eta)} x^{0} \epsilon^{0 i j k} t_{k} \sim \frac{1}{\sinh (\eta)} r^{2} R \epsilon^{i j k} t^{k}
\end{aligned}
$$

so that $\theta^{0 i} \sim r^{2} R t^{i} \gg \theta^{i j}$ at late times $\eta \gg 1$, indicated by $\sim$. Then the first term in (2.8) dominates, and

$$
\theta^{\mu \nu} \sim \frac{r^{2}}{\cosh (\eta)}\left(x^{\mu} t^{\nu}-x^{\nu} t^{\mu}\right)
$$

Note that at $p$, the $t^{\mu}$ generators satisfy

$$
\left\{t^{i}, t^{j}\right\} \stackrel{p}{=}-\frac{1}{r^{2} R^{2}} \theta^{i j}=-\frac{1}{R \sinh (\eta)} \epsilon^{i j k} t^{k}
$$

and $t^{0} \stackrel{p}{=} 0$. Hence in a sense we are considering a $U(\mathfrak{s u}(2))$-valued Yang-Mills-type theory of local space-like translations, however the non-commutativity (via the Poisson bracket) 
leads to novel structures not usually encountered in classical Yang-Mills theory or gravity. Note that the explicit $\epsilon^{i j k}$ in (2.29) implies that parity is broken, ${ }^{8}$ and the sign would be flipped for the doubleton representation $\mathcal{H}_{n}^{\prime}$ with opposite chirality. This is somewhat reminiscent of chiral higher spin theory [15].

Orders of magnitude and asymptotic regime. Now consider the "size" of the various generators. The relations (2.7) lead to the following scale estimates

$$
\begin{aligned}
|t| & \sim r^{-1} \cosh (\eta) \\
\left|\theta^{\mu \nu}\right| & \sim R r \cosh (\eta)=: L_{\mathrm{NC}}^{2}
\end{aligned}
$$

defining the scale of noncommutativity $L_{\mathrm{NC}}$. This gives for $\phi^{(1)}=\phi_{\alpha}(x) t^{\alpha} \in \mathcal{C}^{1}$

$$
\begin{aligned}
\left\{t^{\mu}, \phi^{(1)}\right\} & =\left\{t^{\mu}, \phi_{\alpha}(x)\right\} t^{\alpha}+\phi_{\alpha}\left\{t^{\mu}, t^{\alpha}\right\} \sim \sinh (\eta) \partial \phi_{\alpha} t^{\alpha}-\frac{1}{r^{2} R^{2}} \phi_{\alpha} \theta^{\mu \alpha} \\
& =\frac{1}{r R} O(\sinh (\eta)(\sinh (\eta) R \partial \phi+\phi)) \\
& \sim\left\{t^{\mu}, \phi(x)_{\alpha}\right\} t^{\alpha}\left(1+O\left(\frac{1}{|x| \partial}\right)\right)
\end{aligned}
$$

where $|x|=R \cosh (\eta)$ measures the cosmic time, and $\sim$ indicates $\eta \gg 1$. Hence the derivative term dominates except for extremely low wavelengths $\lambda$, i.e. in the asymptotic regime

$$
L_{\mathrm{NC}} \ll \lambda \ll R \cosh (\eta)
$$

much shorter than the cosmic scale and longer than the scale of noncommutativity. Similarly,

$$
\begin{aligned}
\left\{x^{\mu}, \phi^{(1)}\right\} & =\left\{x^{\mu}, \phi_{\alpha}\right\} t^{\alpha}+\phi_{\alpha}\left\{x^{\mu}, t^{\alpha}\right\}=\theta^{\mu \nu} t^{\alpha} \partial_{\nu} \phi_{\alpha}+\sinh (\eta) \phi_{\mu} \\
& \sim \sinh (\eta)(\sinh (\eta) R \partial \phi+\phi) \\
& \sim\left\{x^{\mu}, \phi(x)_{\alpha}\right\} t^{\alpha}\left(1+O\left(\frac{1}{|x| \partial}\right)\right) .
\end{aligned}
$$

Note that the estimate applies equally to the component in $\mathcal{C}^{0}$ and $\mathcal{C}^{2}$, since $\theta^{\mu \nu} t^{\alpha}$ and $\left[\theta^{\mu \nu} t^{\alpha}\right]_{0}$ are comparable in size. Hence in general we can write

$$
\{\phi, \psi\} \sim\left\{\phi_{\underline{\alpha}}, \psi_{\underline{\beta}}\right\} t^{\underline{\alpha}} t^{\underline{\beta}},
$$

where $\underline{\alpha}$ is a multi-index. This amounts precisely to the projection to $\mathcal{M}(2.26)$ as discussed above, which will be very helpful to extract the leading contributions for physics. Recalling the cosmic FLRW scale parameter (2.11), the ratio of IR and UV scales is

$$
\frac{a(t)^{2}}{L_{N C}^{2}} \sim \frac{R \cosh ^{2}(\eta)}{r} \rightarrow \infty
$$

at late times. Hence space grows indeed much faster than the NC scale, as it should.

\footnotetext{
${ }^{8}$ I would like to thank the anonymous referee for pointing this out.
} 


\subsection{Poisson bracket and reduction to $\mathcal{M}$}

To make the reduction to $\mathcal{M}$ precise, we derive an explicit formula for the Poisson structure with the form

$$
\begin{aligned}
\{f, g\}= & \left\{f, x^{\mu}\right\} M_{\mu}^{\nu}\left\{t_{\nu}, g\right\}-\left\{g, x^{\mu}\right\} M_{\mu}^{\nu}\left\{t_{\nu}, f\right\} \\
& +\left\{f, x^{\mu}\right\} B_{\mu \nu}\left\{x_{\nu}, g\right\}+\left\{g, t_{\mu}\right\} C^{\mu \nu}\left\{t_{\nu}, f\right\}
\end{aligned}
$$

where $B$ and $C$ are antisymmetric. This is not unique due to the constraints

$$
\begin{aligned}
& 0=x^{\mu}\left\{t_{\mu}, .\right\}+t_{\mu}\left\{x^{\mu}, .\right\} \\
& 0=R^{-2} x^{\mu}\left\{x_{\mu}, .\right\}+r^{2} t_{\mu}\left\{t^{\mu}, .\right\} .
\end{aligned}
$$

An explicit realization is given in appendix A.2, which leads to the following formula

$$
\{f, g\}=\left(\mathcal{D}^{\mu} f\right)\left\{t_{\mu}, g\right\}+\left(\varlimsup_{\mu} f\right)\left\{x_{\mu}, g\right\}
$$

where $\mathcal{D}$ and $\varnothing$ are derivations ${ }^{9}$ on $\mathcal{C}$ given by

$$
\begin{aligned}
\varlimsup^{\mu} x^{\rho} & =\eta^{\rho \nu}, & \varlimsup^{\mu} t^{\rho} & =- \\
\mathcal{D}^{\mu} t^{\rho} & =P_{\perp}^{\rho \mu}-r^{2} \cosh ^{-2}(\eta) t^{\rho} t^{\mu}, & \mathcal{D}^{\mu} x^{\rho} & =0 .
\end{aligned}
$$

In particular, $\mathcal{D} \mathcal{C}^{0}=0$ means that its push-forward to $\mathcal{M}$ vanishes, $\Pi_{*} \mathcal{D}=0$. Thus $\mathcal{D}$ are "vertical" derivatives along the local $S^{2}$ fiber. Moreover, we claim that $\precsim=\Pi_{*} \partial$ vanishes on the local $S^{2}$ fiber. To see that, we compute

$$
\mathrm{\partial}^{0} t^{i}=-\frac{1}{R \cosh (\eta)} t^{i}, \quad \varlimsup^{j} t^{i}=0
$$

at the reference point, and note that the first term reproduces the grow of $|t|^{2}=$ $r^{-2} \cosh ^{2}(\eta)=-(r R)^{-2}|x|^{2}$ in time. Hence the push-forward of a Hamiltonian vector field to $\mathcal{M}$ is given by

$$
\{g, .\}_{\mathcal{M}} \equiv \Pi_{*}\{g, .\}=\left\{g, x^{\mu}\right\} \partial_{\mu}
$$

and similarly the push-forward of the Poisson bracket to $\mathcal{M}$ is given by

$$
\{f, g\}_{\mathcal{M}}=\theta^{\mu \nu} \partial_{\mu} f \check{\partial}_{\nu} g
$$

which is verified immediately for $f, g \in \mathcal{C}^{0}$. For late times $\eta \gg 1$, we can replace $\partial$ by the simpler derivative

$$
\partial_{\mu}:=\sinh ^{-1}(\eta)\left\{t_{\mu}, .\right\}
$$

since

$$
\begin{aligned}
& \partial_{\mu} x^{\nu}=\delta_{\mu}^{\nu}, \\
& \partial_{\mu} t^{\nu}=-\frac{1}{r^{2} R^{2}} \sinh ^{-1}(\eta) \theta^{\mu \nu} \sim-\frac{1}{R^{2}} \frac{1}{\cosh ^{2}(\eta)}\left(x^{\mu} t^{\nu}-x^{\nu} t^{\mu}\right)
\end{aligned}
$$

using (2.28), which agrees with $\partial$ at late times. We can then replace (2.42) by

$$
\{f, g\}_{\mathcal{M}} \sim\left(\partial_{\mu} f\right)\left\{x_{\mu}, g\right\} .
$$

This will be used throughout this paper, and we discuss again its range of validity:

\footnotetext{
${ }^{9}$ Note that the present $\partial$ is different from the one used in [31] for $H_{N}^{4}$.
} 
Asymptotic regime. The fact that $\mathcal{D}$ vanishes on $\mathcal{C}^{0}$ is very helpful to organize the higher-spin theory: it leads to the estimate

$$
\left|\mathcal{D} f^{(s)}\right| \leq \frac{s}{|t|}|f|=\frac{r s}{\cosh (\eta)}|f|
$$

for $f=f^{(s)} \in \mathcal{C}^{s}$, where $|f|$ denotes the maximal value of $f$ on the internal $S^{2}$ over the particular point on $\mathcal{M}$. Thus

$$
\begin{aligned}
(\mathcal{D} f)\left\{t_{\mu}, g\right\} & =r O(f \partial g) \\
\left(\partial_{\mu} f\right)\left\{x_{\mu}, g\right\} & =r O(x \cdot \partial f \partial g)
\end{aligned}
$$

for small spin and $\eta \gg 1$, using the abbreviation $x \cdot \partial \equiv R \cosh (\eta) \partial$. Thus

$$
\begin{aligned}
\{g, f\} & =\left\{g, t_{\mu}\right\}\left(\mathcal{D}^{\mu} f\right)+\left\{g, x_{\mu}\right\}\left(\partial_{\mu} f\right) \\
& =\left\{g, x_{\mu}\right\}\left(\partial_{\mu} f\right)\left(1+O\left(\frac{f}{x \cdot \partial f}\right)\right)
\end{aligned}
$$

so that we can use the approximation

$$
\{g, f\} \sim\left\{g, x^{\mu}\right\}\left(\partial_{\mu} f\right)
$$

in the asymptotic regime, i.e. $x \cdot \partial f \gg f$. If both $f$ and $g$ are in the asymptotic regime (2.32), we can write

$$
\{f, g\} \sim \theta^{\mu \nu} \partial_{\mu} g \partial_{\nu} f \sim\{f, g\}_{\mathcal{M}}
$$

in agreement with (2.34). Of course we are free to use any coordinates in $\mathcal{C}^{0}$ here. Thus we have replaced the Poisson structure $\mathbb{C} P^{1,2}$ by a $\mathfrak{h s}$ valued bi-vector field on $\mathcal{M}$, which is its push-forward by the bundle projection as discussed in section 2.1. This reduced Poisson tensor will satisfy the following reduced Jacobi identity

$$
\left\{\{f, g\}_{\mathcal{M}}, h\right\}_{\mathcal{M}}+\left\{\{g, h\}_{\mathcal{M}}, f\right\}_{\mathcal{M}}+\left\{\{h, f\}_{\mathcal{M}}, g\right\}_{\mathcal{M}} \sim 0
$$

which holds exactly on $\mathcal{C}^{0}$ and at least asymptotically for the higher spin components, as long as $x \cdot \partial \gg 1$. This in turn implies the identity (cf. appendix A in [8])

$$
\partial_{\mu}\left(\rho_{M} \theta^{\mu \nu}\right) \sim 0, \quad \rho_{M}={\sqrt{\left|\theta^{\mu \nu}\right|}}^{-1}
$$

with the same qualifications.

\section{$3 \quad$ Higher-spin gauge invariance and Lie derivatives}

\subsection{Gauge transformation of scalar fields}

Consider some scalar field $\phi \in \mathcal{C}$ on $\mathbb{C} P^{1,2}$, which transforms under gauge transformations $\operatorname{as}^{10}$

$$
\delta_{\Lambda} \phi=\{\Lambda, \phi\}=\mathcal{L}_{\xi} \phi, \quad \Lambda=\Lambda^{*} \in \mathcal{C}^{\infty}\left(\mathbb{C} P^{1,2}\right) .
$$

\footnotetext{
${ }^{10}$ This arises from the matrix model gauge transformation $\phi \rightarrow U \phi U^{-1}$ for $\phi \in \operatorname{End}(\mathcal{H})$. For $U=e^{i \Lambda}$, its infinitesimal version is $\phi \rightarrow-i[\Lambda, \phi] \sim\{\Lambda, \phi\}$ for $\Lambda=\Lambda^{\dagger} \in \operatorname{End}(\mathcal{H})$.
} 
This is nothing but the Lie derivative of $\phi \in \mathcal{C}^{\infty}\left(\mathbb{C} P^{1,2}\right)$ along the Hamiltonian vector field $\xi=\{\Lambda,$.$\} . For functions on \mathcal{M}$ i.e. restricted to $\phi \in \mathcal{C}^{0}$, this reduces to

$$
\delta_{\Lambda} \phi=\mathcal{L}_{\xi} \phi=\xi^{\mu} \partial_{\mu} \phi
$$

which is interpreted as Lie derivative along the $\mathfrak{h} \mathfrak{s}$-valued vector field

$$
\xi=\xi^{\mu} \partial_{\mu}, \quad \xi^{\mu}=\left\{\Lambda, x^{\mu}\right\}
$$

on $\mathcal{M}$. E.g. for $\Lambda \in \mathcal{C}^{1}$, its components are

$$
\xi^{\mu}=\xi_{(0)}^{\mu}+\xi_{(2)}^{\mu} \quad \in \mathcal{C}^{0} \oplus \mathcal{C}^{2}
$$

and we can view $\xi_{(0)}^{\mu}=\left\{\Lambda, x^{\mu}\right\}_{0} \in \mathcal{C}^{0}$ as vector field on $\mathcal{M}$ which defines a diffeomorphism

$$
\left[\delta_{\Lambda} \phi\right]_{0}=\mathcal{L}_{\xi_{(0)}} \phi
$$

However one typically cannot get rid of the component $\xi_{(2)}=\left\{\Lambda, x^{\mu}\right\}_{2} \in \mathcal{C}^{2}$, and $\xi$ should be considered as generator of generalized $\mathfrak{h} \mathfrak{s}$-valued diffeomorphisms.

$\mathfrak{h} \mathfrak{s - v a l u e d}$ scalar fields on $\mathcal{M}$. In the asymptotic regime $(2.32)$, the above formula generalizes to arbitrary $\phi \in \mathcal{C}$ as follows:

$$
\begin{aligned}
\delta_{\Lambda} \phi & =\{\Lambda, \phi\} \sim\left\{\Lambda, x^{\mu}\right\} \partial_{\mu} \phi \\
& =\xi^{\mu} \partial_{\mu} \phi=: \overline{\mathcal{L}}_{\xi} \phi .
\end{aligned}
$$

This is interpreted as Lie derivative of a $\mathfrak{h} \mathfrak{s}$-valued function $\phi$ along the $\mathfrak{h} \mathfrak{s}$-valued vector field $\xi$, indicated by $\overline{\mathcal{L}}$. This makes sense as long as $\phi$ and $\xi$ are in the asymptotic regime.

\subsection{Gauge transformation of vector fields}

Now consider some given Hamiltonian vector field $E=\{Z,$.$\} on \mathbb{C} P^{1,2}$, such as the frame discussed below. Assume that $Z$ transforms under a gauge transformation (3.1) as

$$
\delta_{\Lambda} Z=\{\Lambda, Z\} .
$$

Then the associated vector field $E$ transforms as

$$
\begin{aligned}
\left(\delta_{\Lambda} E\right) \phi & =\{\{\Lambda, Z\}, \phi\}=\{\Lambda,\{Z, \phi\}\}-\{Z,\{\Lambda, \phi\}\} \\
& =\left(\mathcal{L}_{\xi} E\right) \phi
\end{aligned}
$$

for any $\phi \in \mathcal{C}$, where $\xi=\{\Lambda,$.$\} . This is precisely the Lie derivative of the vector field E$ along $\xi$, hence

$$
\delta_{\Lambda} E=\mathcal{L}_{\xi} E .
$$

This will give the transformation of the frame in (4.2), and it extends to higher-rank tensor fields as

$$
\delta_{\Lambda}\left(E \otimes E^{\prime}\right)=\left(\mathcal{L}_{\xi} E\right) \otimes E^{\prime}+E \otimes \mathcal{L}_{\xi} E^{\prime}=\mathcal{L}_{\xi}\left(E \otimes E^{\prime}\right)
$$

which will apply to the metric tensor. We also recall that the Lie derivative of the Poisson bi-vector field along any Hamiltonian vector field $\xi$ on $\mathbb{C} P^{1,2}$ vanishes $\mathcal{L}_{\xi}\{.,\}=$.0 , due to the Jacobi identity. 
Gauge transformations and $\mathfrak{h s}$-valued Lie derivative on $\mathcal{M}$. Now consider the same from the 4-dimensional point of view. As discussed in section 2.1, the push-forward of the Hamiltonian vector field $E=\{Z,$.$\} to \mathcal{M}$ for $Z \in \mathcal{C}$ defines a $\mathfrak{h s - v a l u e d}$ vector field $E^{\mu} \partial_{\mu}$ on $\mathcal{M}$, with components

$$
E^{\mu}:=\left\{Z, x^{\mu}\right\}
$$

Under gauge transformations $\delta_{\Lambda} Z=\{\Lambda, Z\}$, we can still use the first line in (3.8),

$$
\left(\delta_{\Lambda} E\right) \phi=\{\Lambda,\{Z, \phi\}\}-\{Z,\{\Lambda, \phi\}\}
$$

At this point it is more transparent to use coordinates $x^{\mu}$ on $\mathcal{M}$, and the above becomes

$$
\begin{aligned}
\delta_{\Lambda} E^{\mu} & =\left\{\Lambda, E^{\mu}\right\}-\left\{Z, \xi^{\mu}\right\} \\
& \sim \xi^{\rho} \partial_{\rho} E^{\mu}-\left\{Z, x^{\rho}\right\} \partial_{\rho} \xi^{\mu} \\
& =\xi^{\rho} \partial_{\rho} E^{\mu}-E^{\rho} \partial_{\rho} \xi^{\mu} \\
& =: \overline{\mathcal{L}}_{\xi} E^{\mu}
\end{aligned}
$$

(for $\phi=x^{\mu}$ ), using the approximation (2.49) in the second line in the asymptotic regime. This has the standard form of a Lie derivative along a field $\xi^{\mu}=\left\{\Lambda, x^{\mu}\right\}$, and constitutes our "working definition" of a $\mathfrak{h} \mathfrak{s}$-valued Lie derivative $\overline{\mathcal{L}}$ on $\mathcal{M}$, generalizing (3.6). Remember that both $E^{\mu}$ and $\xi^{\mu}$ have $\mathfrak{h} \mathfrak{s}$ components.

The above considerations provide a 4-dimensional geometrical interpretation of the emergent diffeomorphism invariance and its higher spin extension, which arise ${ }^{11}$ from Hamiltonian vector fields on $\mathbb{C} P^{1,2}$. Since these preserve the symplectic volume on $\mathbb{C} P^{1,2}$, the resulting $\mathfrak{h} \mathfrak{s}$ diffeomorphisms on $\mathcal{M}$ should be volume-preserving in some sense also from the 4-dimensional point of view. This is indeed the case, as elaborated in appendix A.1.

\section{Kinetic action, frame and effective metric on $\mathcal{M}$}

Now we want to understand the effective metric in the matrix model in the non-linear regime. To keep the discussion simple, we focus on the kinetic term for a scalar field $\phi$ in the matrix model,

$$
S[\phi]=\operatorname{Tr}\left[Z^{\dot{\alpha}}, \phi\right]\left[Z_{\dot{\alpha}}, \phi\right]=-\operatorname{Tr} \phi \square \phi, \quad \dot{\alpha}=0, \ldots, 3
$$

on a background given by some solution $Z_{\dot{\alpha}}$ of (2.3). Such scalar fields arise as transversal brane fluctuation $\phi \equiv Z_{b}, b=4, \ldots, 9$. The effective metric is encoded in the d'Alembertian $\square$, and will therefore govern all fluctuations in the model. We study the above action in the semi-classical limit from two different points of view.

$\mathbb{C} P^{1,2}$ point of view. Consider a function $\phi$ on $\mathbb{C} P^{1,2}$. The background $Z_{\dot{\alpha}}$ defines a frame

$$
E_{\dot{\alpha}}[\phi]:=\left\{Z_{\dot{\alpha}}, \phi\right\}, \quad \dot{\alpha}=0, \ldots, 3
$$

\footnotetext{
${ }^{11}$ This generalizes analogous results in $[24]$ in a dimensionally reduced setting.
} 
which are derivations on $\mathcal{C}=\mathcal{C}^{\infty}\left(\mathbb{C} P^{1,2}\right)$. Frame indices will always be dotted Greek letters $\dot{\alpha}, \dot{\beta}, \ldots$, which transform under the global $\mathrm{SO}(3,1)$. This defines a metric as a bi-vector field

$$
\gamma[\phi, \psi]:=\eta^{\dot{\alpha} \dot{\beta}} E_{\dot{\alpha}}[\phi] E_{\dot{\beta}}[\psi]
$$

and the action (4.1) can be written in the semi-classical limit as ${ }^{12}$

$$
\begin{aligned}
S[\phi] & =\operatorname{Tr}\left[Z^{\dot{\alpha}}, \phi\right]\left[Z_{\dot{\alpha}}, \phi\right] \sim-\int_{\mathbb{C} P^{1,2}} \omega^{\wedge 3}\left\{Z^{\dot{\alpha}}, \phi\right\}\left\{Z_{\dot{\alpha}}, \phi\right\} \\
& =-\int_{\mathbb{C} P^{1,2}} \mathrm{~d}^{6} z \frac{1}{\sqrt{\left|\theta^{A B}\right|}} \gamma^{A B} \partial_{A} \phi \partial_{B} \phi
\end{aligned}
$$

where e.g. $y^{A}=\left(x^{\mu}, \vartheta, \varphi\right)$ are coordinates on $\mathbb{C} P^{1,2}$. Here the coordinate form of the frame is

$$
E_{\dot{\alpha}}^{A}:=\left\{Z_{\dot{\alpha}}, y^{A}\right\}, \quad \gamma^{A B}=\eta^{\dot{\alpha} \dot{\beta}} E_{\dot{\alpha}}^{A} E_{\dot{\beta}}^{B}
$$

such that $E_{\dot{\alpha}}[\phi]=E_{\dot{\alpha}}^{A} \partial_{A} \phi$ for $\phi \in \mathcal{C}$, and the $\mathrm{SO}(4,2)$-invariant symplectic volume form on $\mathbb{C} P^{1,2}$ is

$$
\omega^{\wedge 3}=\Omega_{A_{1} \ldots A_{6}} d y^{A_{1}} \ldots d y^{A_{6}}=\frac{1}{\sqrt{\left|\theta^{A B}\right|}} d y^{1} \ldots d y^{6} .
$$

However, this does not properly reflect the structure of $\mathbb{C} P^{1,2}$ as $S^{2}$ bundle over $\mathcal{M}$, and we will rewrite it in a way which is more transparent from the 4-dimensional point of view.

3+1-dimensional point of view on $\mathcal{M}$. Now consider the reduction (more precisely the push-forward) of the same configuration to $\mathcal{M}$. This applies automatically if $\phi$ is in the asymptotic regime. Then

$$
E_{\dot{\alpha}}[\phi] \sim E_{\dot{\alpha}}{ }^{\mu} \partial_{\mu} \phi, \quad E_{\dot{\alpha}}{ }^{\mu}:=\left\{Z_{\dot{\alpha}}, x^{\mu}\right\}
$$

is a $\mathfrak{h} \mathfrak{s}$-valued frame on $\mathcal{M}$, which is an invertible $\mathcal{C}$-valued $4 \times 4$ matrix. Instead of the Cartesian coordinates $x^{\mu}$ we could use any other coordinates on $\mathcal{M}$. We can write the volume form (4.6) as

$$
\omega^{\wedge 3}=\rho_{M} d x^{0} \ldots d x^{3} \Omega_{2}, \quad \rho_{M}=\sinh ^{-1}(\eta)
$$

where $\Omega_{2}$ is the volume form of the unit 2-sphere corresponding to the local fiber, and $\rho_{M}$ is the corresponding 4-density. The explicit form is easily obtained as the unique $\mathrm{SO}(4,1)$-invariant volume on $H^{4}$, cf. [32]. Then the action reduces to

$$
S[\phi] \sim-\int_{\mathcal{M}^{3,1}} d x_{0} \ldots d x_{3} \rho_{M} \gamma^{\mu \nu} \partial_{\mu} \phi \partial_{\nu} \phi=-\int_{\mathcal{M}^{3,1}} \mathrm{~d}^{4} x \sqrt{\left|G_{\mu \nu}\right|} G^{\mu \nu} \partial_{\mu} \varphi \partial_{\nu} \varphi
$$

absorbing some dimensionful constants in $\varphi \sim \phi$. Here

$$
\gamma^{\mu \nu}=\eta^{\dot{\alpha} \dot{\beta}} E_{\dot{\alpha}}{ }^{\mu} E_{\dot{\beta}}{ }^{\nu}, \quad G^{\mu \nu}:=\frac{1}{\rho^{2}} \gamma^{\mu \nu}
$$

\footnotetext{
${ }^{12}$ The 6 -dimensional action could be rewritten in covariant form using an effective metric along the lines of [8], but we refrain from doing so because we want to emphasize the 4-dimensional point of view.
} 


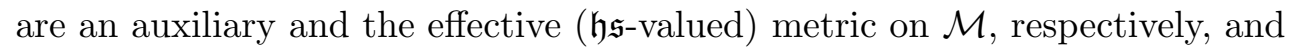

$$
\rho^{2}=\rho_{M} \sqrt{\mid \gamma^{\mu \nu \mid}}
$$

is a scalar field (rather than a density). One can also introduce a densitized vielbein which directly gives the effective metric,

$$
\mathcal{E}_{\dot{\alpha}}{ }^{\mu}=\rho^{-1} E_{\dot{\alpha}}{ }^{\mu}, \quad G^{\mu \nu}=\eta^{\dot{\alpha} \dot{\beta}} \mathcal{E}_{\dot{\alpha}}{ }^{\mu} \mathcal{E}_{\dot{\beta}}{ }^{\nu} .
$$

The last form of (4.9) is indeed $\mathfrak{h} \mathfrak{s}$ covariant, in the sense discussed below. This is the dominant contribution for fields $\phi \in \mathcal{C}^{s}$ in the asymptotic regime. The metric $G^{\mu \nu}$ indeed governs the d'Alembertian (2.20),

$$
\square=-\left\{Z^{\dot{\alpha}},\left\{Z_{\dot{\alpha}}, .\right\}\right\}=\rho^{2} \square_{G}
$$

as shown in Lemma (5.1). At the linearized level and for scalar fields $\phi \in \mathcal{C}^{0}$, only the scalar component $\left[G^{\mu \nu}\right]_{0} \in \mathcal{C}^{0}$ contributes.

For the background solution $\bar{Z}_{\dot{\alpha}}=T_{\dot{\alpha}}=R^{-1} \mathcal{M}_{\dot{\alpha} 4}$ (2.19), we obtain in Cartesian coordinates

$$
\begin{aligned}
\bar{E}_{\dot{\alpha}}^{\mu} & =\sinh (\eta) \delta_{\dot{\alpha}}^{\mu}, & \bar{\gamma}^{\mu \nu} & =\sinh ^{2}(\eta) \eta^{\mu \nu} \\
\bar{G}^{\mu \nu} & =\sinh ^{-3}(\eta) \bar{\gamma}^{\mu \nu}=\sinh ^{-1}(\eta) \eta^{\mu \nu}, & \bar{\rho}^{2} & =\sinh ^{3}(\eta) .
\end{aligned}
$$

Gauge transformation of the frame. Consider first the $\mathbb{C} P^{1,2}$ point of view. Under a gauge transformation (3.1), the background transforms as

$$
\delta_{\Lambda} Z^{\dot{\alpha}}=\left\{\Lambda, Z^{\dot{\alpha}}\right\}
$$

and the associated vector fields $E_{\dot{\alpha}}$ on $\mathbb{C} P^{1,2}$ transform as

$$
\delta_{\Lambda} E_{\dot{\alpha}}=\mathcal{L}_{\xi} E_{\dot{\alpha}}
$$

using (3.9), where $\xi=\{\Lambda,$.$\} . In local coordinates y^{A}$, this gives the coordinate expression

$$
\delta_{\Lambda} E_{\dot{\alpha}}^{A}=\xi^{B} \partial_{B} E_{\dot{\alpha}}^{A}-E_{\dot{\alpha}}^{B} \partial_{B} \xi^{A}=\mathcal{L}_{\xi} E_{\dot{\alpha}}^{A}
$$

and we obtain

$$
\delta_{\Lambda} \gamma^{A B}=\delta_{\Lambda}\left(E_{\dot{\alpha}}^{A} E_{\dot{\beta}}^{B} \eta^{\dot{\alpha} \dot{\beta}}\right)=\mathcal{L}_{\xi} \gamma^{A B} .
$$

Reduction to $\mathcal{M}$. Now consider the gauge transformations of the frame for $\mathfrak{h} \mathfrak{s -}$ valued functions on $\mathcal{M}$, in the asymptotic regime where all fields are in the asymptotic regime (2.32). Then we can use (3.13),

$$
\delta_{\Lambda} E_{\dot{\alpha}}^{\mu} \sim \overline{\mathcal{L}}_{\xi} E_{\dot{\alpha}}{ }^{\mu}
$$

where $\xi^{\nu}=\left\{\Lambda, x^{\nu}\right\}$ is a $\mathfrak{h} \mathfrak{s}$-valued vector field, and $E_{\dot{\alpha}}^{\mu}$ is the $\mathfrak{h} \mathfrak{s}$-valued frame (4.7) on $\mathcal{M}$. In particular, this implies

$$
\delta_{\Lambda} \gamma^{\mu \nu} \sim \overline{\mathcal{L}}_{\xi} \gamma^{\mu \nu}=\nabla_{(\gamma)}^{\mu} \xi^{\nu}+\nabla_{(\gamma)}^{\nu} \xi^{\mu}
$$


where $\nabla_{(\gamma)}$ is the Levi-Civita connection corresponding to $\gamma^{\mu \nu}$. We would like to generalize this to the effective metric $G^{\mu \nu}$ including the conformal factor. To see this, observe that the invariance of the symplectic form $\mathcal{L}_{\xi} \omega=0$ implies

$$
\delta_{\Lambda} \rho^{4} \sim \overline{\mathcal{L}}_{\xi} \rho^{4}
$$

for

$$
\rho^{4}:=\gamma^{\mu_{0} \nu_{0}} \ldots \gamma^{\mu_{3} \nu_{3}} \Omega_{\mu_{0} \ldots \mu_{3}} \Omega_{\nu_{0} \ldots \nu_{3}}=\left|\gamma^{\mu \nu}\right|\left|\omega_{\mu \nu}\right|=\left|\gamma^{\mu \nu}\right| \rho_{M}^{2},
$$

where

$$
\Omega_{\mu_{0} \ldots \mu_{3}}:=\left(\omega^{\wedge 2}\right)_{\mu_{0} \ldots \mu_{3}}, \quad \Omega_{\mu_{0} \ldots \mu_{3}} \varepsilon^{\mu_{0} \ldots \mu_{3}}=\sqrt{\left|\omega_{\mu \nu}\right|}=\rho_{M}
$$

is the effective volume form $(4.8)$ on $\mathcal{M}$. Thus $\rho^{2}$ coincides with the conformal factor for the effective metric (4.10), and we obtain

$$
\delta_{\Lambda} G^{\mu \nu} \sim \overline{\mathcal{L}}_{\xi} G^{\mu \nu} \sim \nabla_{(G)}^{\mu} \xi^{\nu}+\nabla_{(G)}^{\nu} \xi^{\mu}
$$

for the effective metric in the asymptotic regime. Here $\nabla_{(G)}$ is the Levi-Civita connection corresponding to $G^{\mu \nu}$.

To summarize, the gauge invariance arising from symplectomorphisms on $\mathbb{C} P^{1,2}$ leads to an emergent higher-spin symmetry in 4 dimensions. This is achieved by considering $E_{\dot{\alpha}}{ }^{\mu}, G^{\mu \nu}$ and $\xi^{\mu}$ as $\mathfrak{h} \mathfrak{s - v a l u e d}$ vector fields on $\mathcal{M}^{3,1}$, and (4.24) should be understood in this higher spin sense. In the linearized regime for $s=1$, this reduces to the standard formulas for volume-preserving diffeomorphisms in 4 dimensions [12].

\section{Geometric description of the non-linear regime}

In this section, we develop a geometric formalism based on a higher-spin generalization of the Weitzenböck connection and torsion. We will work in the asymptotic regime (2.32), using the 4-dimensional point of view developed above.

\subsection{Weitzenböck connection and torsion}

The fundamental degrees of freedom of the matrix model is the background $Z_{\dot{\alpha}}$ and its associated vielbein $E_{\dot{\alpha}}=\left\{Z_{\dot{\alpha}},.\right\}$. It is then natural to define a connection which respects the vielbein,

$$
0=\nabla_{\dot{\gamma}} E_{\dot{\alpha}}^{\mu}=E_{\dot{\gamma}}\left[E_{\dot{\alpha}}{ }^{\mu}\right]+\Gamma_{\dot{\gamma} \rho}{ }^{\mu} E_{\dot{\alpha}}{ }^{\rho}
$$

analogous to the Weitzenböck connection [33], along $E_{\dot{\gamma}}$. This can always be solved as

$$
\Gamma_{\dot{\gamma} \dot{\alpha}}^{\mu}:=-E_{\dot{\gamma}}\left[E_{\dot{\alpha}}{ }^{\mu}\right]=\Gamma_{\dot{\gamma} \rho}{ }^{\mu} E_{\dot{\alpha}}{ }^{\rho} \quad \in \mathcal{C}
$$

provided $E_{\dot{\alpha}}^{\rho}$ is an invertible matrix taking values in $\mathcal{C}$. In the perturbative regime, the most significant contribution should be the $\mathcal{C}^{0}$ components of $E_{\dot{\alpha}}{ }^{\mu}$ and $\Gamma_{\dot{\gamma} \rho}{ }^{\mu}$, accompanied by some higher-spin contributions. In local coordinates $y^{\mu}$, this is

$$
\partial_{\nu}{E_{\dot{\alpha}}}^{\mu}=-\Gamma_{\nu \rho}{ }^{\mu} E_{\dot{\alpha}}{ }^{\rho}
$$


where we define the derivation ${ }^{13}$

$$
\partial_{\nu}:=E^{\dot{\alpha}}{ }_{\nu}\left\{Z_{\dot{\alpha}}, \cdot\right\}, \quad \partial_{\nu} y^{\mu}=\delta_{\nu}^{\mu} .
$$

The inverse vierbein is defined as usual

$$
\begin{array}{ll}
E^{\dot{\alpha}}{ }_{\mu} E_{\dot{\beta}}{ }^{\mu}=\delta_{\dot{\beta}}^{\dot{\alpha}}, & \gamma^{\mu \nu}=\eta^{\dot{\alpha} \dot{\beta}} E_{\dot{\alpha}}{ }^{\mu} E_{\dot{\beta}}{ }^{\nu}, \\
E^{\dot{\alpha}}{ }_{\nu} E_{\dot{\alpha}}{ }^{\mu}=\delta_{\nu}^{\mu}, & \eta_{\dot{\alpha} \dot{\beta}}=E_{\dot{\alpha}}{ }^{\mu} E_{\dot{\beta}}{ }^{\nu} \gamma_{\mu \nu} .
\end{array}
$$

This connection is automatically compatible with the metric $\gamma^{\mu \nu}$,

$$
\nabla \gamma^{\mu \nu}=\eta^{\dot{\alpha} \dot{\beta}}\left(\nabla E_{\dot{\alpha}}{ }^{\mu} E_{\dot{\beta}}{ }^{\nu}+E_{\dot{\alpha}}{ }^{\mu} \nabla E_{\dot{\beta}}{ }^{\nu}\right)=0 \text {. }
$$

For any $\mathfrak{h} \mathfrak{s - v a l u e d}$ vector field $V^{\mu}$ on $\mathcal{M}$, we can then define the covariant derivative as

$$
\nabla_{\mu} V^{\nu}=\partial_{\mu} V^{\nu}+\Gamma_{\mu \rho}^{\nu} V^{\rho}
$$

This connection is flat ${ }^{14}$ since the frame is parallel, $\nabla E_{\dot{\beta}}=0$. However it typically has torsion,

$$
T[X, Y]=\nabla_{X} Y-\nabla_{Y} X-[X, Y]
$$

which for the frame can be computed as

$$
\begin{aligned}
T_{\dot{\gamma} \dot{\beta}} & \equiv T\left[E_{\dot{\gamma}}, E_{\dot{\beta}}\right]=\nabla_{\dot{\gamma}} E_{\dot{\beta}}-\nabla_{\dot{\beta}} E_{\dot{\gamma}}-\left[E_{\dot{\gamma}}, E_{\dot{\beta}}\right] \\
& =-\left[E_{\dot{\gamma}}, E_{\dot{\beta}}\right] \equiv-\left\{Z_{\dot{\gamma}},\left\{Z_{\dot{\beta}}, .\right\}\right\}+\left\{Z_{\dot{\beta}},\left\{Z_{\dot{\gamma}}, .\right\}\right\} \\
& =\left\{\hat{\Theta}_{\dot{\gamma} \dot{\beta}}, .\right\}, \\
T_{\dot{\alpha} \dot{\beta}}{ }^{\mu} & :=\left\{\hat{\Theta}_{\dot{\alpha} \dot{\beta}}, y^{\mu}\right\}, \quad \hat{\Theta}_{\dot{\alpha} \dot{\beta}}:=-\left\{Z_{\dot{\alpha}}, Z_{\dot{\beta}}\right\}
\end{aligned}
$$

using the Jacobi identity. Due to Lemma 2.1, $T_{\dot{\alpha} \dot{\beta}}{ }^{\mu}$ fully captures the noncommutative field strength $\hat{\Theta}_{\dot{\alpha} \dot{\beta}}$. Thus torsion encodes the quantum structure of space-time, and it is the semi-classical shadow of it. This is the key to understand the matrix model in terms of gravity, ${ }^{15}$ and the equations of motion of the model will be re-formulated in terms of torsion below. For a perturbed cosmic background $Z_{\dot{\alpha}}=t_{\dot{\alpha}}+\mathcal{A}_{\dot{\alpha}}$, we have

$$
\hat{\Theta}_{\dot{\alpha} \dot{\beta}}=\frac{1}{r^{2} R^{2}} \theta_{\dot{\alpha} \dot{\beta}}-\left(\left\{t_{\dot{\alpha}}, \mathcal{A}_{\dot{\beta}}\right\}-\left\{t_{\dot{\beta}}, \mathcal{A}_{\dot{\alpha}}\right\}+\left\{\mathcal{A}_{\dot{\alpha}}, \mathcal{A}_{\dot{\beta}}\right\}\right)
$$

\footnotetext{
${ }^{13}$ For the background $Z_{\dot{\alpha}}=t_{\dot{\alpha}}$, the $\partial_{\nu}$ agrees with $\partial_{\nu}$ and $(2.43)$ on $\mathcal{C}^{0}$, and possible differences on $\mathfrak{h} \mathfrak{s}$ are negligible in the asymptotic regime.

${ }^{14}$ The curvature on $\mathcal{M}$ is defined as usual by $\mathcal{R}_{\dot{\alpha}, \dot{\beta}}\left[E_{\dot{\gamma}}\right]:=\left[\nabla_{\dot{\alpha}}, \nabla_{\dot{\beta}}\right] E_{\dot{\gamma}}-\nabla_{\left[E_{\dot{\alpha}}, E_{\dot{\beta}}\right]} E_{\dot{\gamma}}=0$ in the asymptotic regime, where $\left[E_{\dot{\alpha}}, E_{\dot{\beta}}\right]$ is a linear combination of the $E_{\dot{\gamma}}$. However, there is no fully noncommutative version.

${ }^{15}$ The role of torsion in a dimensionally reduced noncommutative gauge theory as related to gravity was already pointed out in [24], however the specifics are different. Torsion arises in (5.9) as derivative of the NC field strength, unlike in [24]. Also, the action in previous work is typically given by a contraction of torsion, which is not the case here. See also e.g. [34] for other work related to torsion in a similar context.
} 
so that $\hat{\Theta}_{\dot{\alpha} \dot{\beta}} \in \mathcal{C}^{1}$ up to higher-spin corrections. More explicitly, the torsion tensor is

$$
\begin{aligned}
T_{\dot{\alpha} \dot{\beta}}{ }^{\mu} & =\nabla_{\dot{\alpha}} E_{\dot{\beta}}{ }^{\mu}-\nabla_{\dot{\beta}} E_{\dot{\alpha}}{ }^{\mu}-\left[E_{\dot{\alpha}}, E_{\dot{\beta}}\right]^{\mu} \\
& =E_{\dot{\alpha}}\left[E_{\dot{\beta}}{ }^{\mu}\right]-E_{\dot{\beta}}\left[E_{\dot{\alpha}}{ }^{\mu}\right]+\Gamma_{\dot{\alpha} \rho}{ }^{\mu} E_{\dot{\beta}}{ }^{\rho}-\Gamma_{\dot{\beta} \rho}{ }^{\mu} E_{\dot{\alpha}}{ }^{\rho}-\left[E_{\dot{\alpha}}, E_{\dot{\beta}}\right]^{\mu} \\
& =\Gamma_{\dot{\alpha} \rho}{ }^{\mu} E_{\dot{\beta}}{ }^{\rho}-\Gamma_{\dot{\beta} \rho}{ }^{\mu} E_{\dot{\alpha}}{ }^{\rho}=\Gamma_{\dot{\alpha} \dot{\beta}}{ }^{\mu}-\Gamma_{\dot{\beta} \dot{\alpha}}{ }^{\mu} \\
T_{\mu \nu}{ }^{\rho} & =\Gamma_{\mu \nu}{ }^{\rho}-\Gamma_{\nu \mu}{ }^{\rho}
\end{aligned}
$$

using the Jacobi identity $E_{\dot{\alpha}}\left[E_{\dot{\beta}}^{\mu}\right]-E_{\dot{\beta}}\left[E_{\dot{\alpha}}^{\mu}\right] \equiv\left[E_{\dot{\alpha}}, E_{\dot{\beta}}\right]^{\mu}$. The gauge transformation of the torsion tensor in the asymptotic regime is obtained again from (3.13),

$$
\begin{aligned}
\delta_{\Lambda} \hat{\Theta}_{\dot{\alpha} \dot{\beta}} & =\left\{\Lambda, \hat{\Theta}_{\dot{\alpha} \dot{\beta}}\right\}, \\
\delta_{\Lambda} T_{\dot{\alpha} \dot{\beta}}{ }^{\mu} & \sim \xi^{\nu} \partial_{\nu} T_{\dot{\alpha} \dot{\beta}}{ }^{\mu}-T_{\dot{\alpha} \dot{\beta}}{ }^{\nu} \partial_{\nu} \xi^{\mu}=\overline{\mathcal{L}}_{\xi} T_{\dot{\alpha} \dot{\beta}}{ }^{\mu}
\end{aligned}
$$

where $\xi^{\nu}=\left\{\Lambda, x^{\nu}\right\}$ is a $\mathfrak{h} \mathfrak{s}$-valued vector field on $\mathcal{M}$. Together with (4.19) this implies

$$
\delta_{\Lambda} T_{\rho \sigma}{ }^{\mu} \sim \overline{\mathcal{L}}_{\xi} T_{\rho \sigma}^{\mu}
$$

and similarly for the effective frame using (4.21). Hence torsion transforms as a covariant tensor, just like the metric.

Relation with the effective Levi-Civita connection. Now consider the Levi-Civita connection $\nabla^{(\gamma)}$ for the metric $\gamma^{\mu \nu}$, which is obtained as usual from the Christoffel symbols

$$
\begin{aligned}
\Gamma_{\mu \nu}^{(\gamma)}{ }^{\sigma} & =\frac{1}{2} \gamma^{\sigma \rho}\left(\partial_{\mu} \gamma_{\rho \nu}+\partial_{\nu} \gamma_{\rho \mu}-\partial_{\rho} \gamma_{\mu \nu}\right) \\
& =\frac{1}{2} \gamma^{\sigma \rho}\left(\Gamma_{\mu \rho \nu}+\Gamma_{\mu \nu \rho}+\Gamma_{\nu \rho \mu}+\Gamma_{\nu \mu \rho}-\Gamma_{\rho \mu \nu}-\Gamma_{\rho \nu \mu}\right) \\
& =\Gamma_{\mu \nu}{ }^{\sigma}-K_{\mu \nu}{ }^{\sigma} .
\end{aligned}
$$

Here

$$
K_{\mu \nu}^{\sigma}=\frac{1}{2}\left(T_{\mu \nu}^{\sigma}+T_{\mu \nu}^{\sigma}-T_{\nu}^{\sigma}{ }_{\mu}\right)=-K_{\mu \nu}^{\sigma} \quad \in \mathcal{C}
$$

is (a higher-spin analog of) the contorsion of the basic Weitzenböck connection, which is antisymmetric in $\nu \sigma$. Therefore

$$
\begin{aligned}
\Gamma_{\mu \nu}{ }^{\rho} & =\Gamma_{\mu \nu}^{(\gamma)}{ }^{\rho}+K_{\mu \nu}{ }^{\rho} \\
\nabla_{\mu} V^{\nu} & =\nabla_{\mu}^{(\gamma)} V^{\nu}+K_{\mu \rho}{ }^{\nu} V^{\rho} .
\end{aligned}
$$

Similarly, the Levi-Civita connection $\nabla^{(G)}$ for the effective metric $G^{\mu \nu}$ is obtained as

$$
\begin{aligned}
\Gamma_{\mu \nu}^{(G)} & =\frac{1}{2} G^{\sigma \rho}\left(\partial_{\mu} G_{\rho \nu}+\partial_{\nu} G_{\rho \mu}-\partial_{\rho} G_{\mu \nu}\right) \\
& =\frac{1}{2} \rho^{-2}\left(\delta_{\nu}^{\sigma} \partial_{\mu} \rho^{2}+\delta_{\mu}^{\sigma} \partial_{\nu} \rho^{2}-\gamma_{\mu \nu} \gamma^{\sigma \rho} \partial_{\rho} \rho^{2}\right)+\frac{1}{2} \gamma^{\sigma \rho}\left(\partial_{\mu} \gamma_{\rho \nu}+\partial_{\nu} \gamma_{\rho \mu}-\partial_{\rho} \gamma_{\mu \nu}\right)
\end{aligned}
$$

which together with the above gives

$$
\Gamma_{\mu \nu}^{(G)}{ }^{\sigma}=\tilde{\Gamma}_{\mu \nu}{ }^{\sigma}-\mathcal{K}_{\mu \nu}{ }^{\sigma}=\Gamma_{\mu \nu}{ }^{\sigma}+\delta_{\nu}^{\sigma} \rho^{-1} \partial_{\mu} \rho-\mathcal{K}_{\mu \nu}{ }^{\sigma}
$$


Here

$$
\begin{aligned}
& \tilde{\Gamma}_{\mu \nu}{ }^{\sigma}:=\Gamma_{\mu \nu}{ }^{\sigma}+\delta_{\nu}^{\sigma} \rho^{-1} \partial_{\mu} \rho, \\
& \mathcal{K}_{\mu \nu}{ }^{\sigma}=K_{\mu \nu}{ }^{\sigma}+\left(G_{\mu \nu} \rho^{-1} \partial^{\sigma} \rho-\delta_{\mu}^{\sigma} \rho^{-1} \partial_{\nu} \rho\right)=-\mathcal{K}_{\mu}{ }^{\sigma}{ }_{\nu}
\end{aligned}
$$

will be recognized below as Weitzenböck connection and contorsion of the effective frame, and accordingly the indices should be raised and lowered with $G^{\mu \nu}$. To avoid any confusion with the two metrics $\gamma^{\mu \nu}$ and $G^{\mu \nu}$, all connection and (con)torsion symbols will be written with two lower and one upper index, where no ambiguity arises. This allows to rewrite the effective Levi-Civita connection in terms of the Weitzenböck connection and the torsion, which amounts to the simple rule for the covariant derivatives

$$
\begin{aligned}
& \nabla_{\mu}^{(G)} V^{\sigma}=\nabla_{\mu} V^{\sigma}-\mathcal{K}_{\mu \nu}{ }^{\sigma} V^{\nu}+\rho^{-1} \partial_{\mu} \rho V^{\sigma} \\
& \nabla_{\mu}^{(G)} V_{\sigma}=\nabla_{\mu} V_{\sigma}+\mathcal{K}_{\mu \sigma}{ }^{\nu} V_{\nu}-\rho^{-1} \partial_{\mu} \rho V_{\sigma}
\end{aligned}
$$

and similarly for higher-rank tensors.

Effective (rescaled) frame. The rescaled or effective frame (4.12) for the effective metric $^{16}$

$$
\mathcal{E}_{\dot{\alpha}}{ }^{\mu}=\frac{1}{\rho} E_{\dot{\alpha}}{ }^{\mu}=\frac{1}{\rho}\left\{Z_{\dot{\alpha}}, y^{\mu}\right\}
$$

gives rise to an associated Weitzenböck connection which is compatible with the effective metric,

$$
\tilde{\nabla} \mathcal{E}_{\dot{\alpha}}{ }^{\mu}=0=\tilde{\nabla} G^{\mu \nu} .
$$

One must be very careful with the frame indices, since there are two different frames in the game. It is therefore safer to use the coordinate form. Then

$$
\begin{aligned}
& \tilde{\Gamma}_{\nu \dot{\alpha}}{ }^{\mu}=-\partial_{\nu} \mathcal{E}_{\dot{\alpha}}{ }^{\mu}=-\partial_{\nu}\left(\rho^{-1} E_{\dot{\alpha}}{ }^{\mu}\right)=\rho^{-1} \Gamma_{\nu \dot{\alpha}}{ }^{\mu}+\rho^{-1} \partial_{\nu} \rho \mathcal{E}_{\dot{\alpha}}{ }^{\mu}=: \tilde{\Gamma}_{\nu \rho}{ }^{\mu} \mathcal{E}_{\dot{\alpha}}{ }^{\rho} \\
& \tilde{\Gamma}_{\nu \sigma}{ }^{\mu}=\Gamma_{\nu \sigma}{ }^{\mu}+\rho^{-1} \delta_{\sigma}^{\mu} \partial_{\nu} \rho .
\end{aligned}
$$

For the covariant derivatives, this amounts to the simple rule

$$
\begin{aligned}
& \tilde{\nabla}_{\mu} V^{\sigma}=\nabla_{\mu} V^{\sigma}+\rho^{-1} \partial_{\mu} \rho V^{\sigma} \\
& \tilde{\nabla}_{\mu} V_{\sigma}=\nabla_{\mu} V_{\sigma}-\rho^{-1} \partial_{\mu} \rho V_{\sigma}
\end{aligned}
$$

and similarly for higher-rank tensors. Then the torsion tensor is

$$
\mathcal{T}_{\mu \nu}{ }^{\sigma}=\tilde{\Gamma}_{\mu \nu}{ }^{\sigma}-\tilde{\Gamma}_{\nu \mu}{ }^{\sigma}=T_{\mu \nu}{ }^{\sigma}+\rho^{-1}\left(\delta_{\nu}^{\sigma} \partial_{\mu} \rho-\delta_{\mu}^{\sigma} \partial_{\nu} \rho\right)
$$

and the effective contorsion is related to that of the basic frame as follows

$$
\mathcal{K}_{\mu \nu \sigma}=K_{\mu \nu \sigma}+\rho^{-1}\left(G_{\mu \nu} \partial_{\sigma} \rho-G_{\mu \sigma} \partial_{\nu} \rho\right)=-\mathcal{K}_{\mu \sigma \nu}
$$

in complete agreement with (5.19). Calligraphic fonts (or a tilde) indicate the rescaled frame.

\footnotetext{
${ }^{16}$ Note that $\mathcal{E}_{\dot{\alpha}}{ }^{\mu}$ is not the Hamiltonian vector field associated to $\frac{1}{\rho} \mathcal{E}_{\dot{\alpha}}$.
} 
(Harmonic) normal coordinates. We will denote coordinates around some point $p \in$ $\mathcal{M}$ as normal coordinates at $p$ if the Christoffel symbols vanish at $p$,

$$
\left.\Gamma_{\nu \sigma}^{(G)}{ }^{\mu}\right|_{p}=0 .
$$

Then the Weitzenböck connection coincides with the contorsion due to (5.18),

$$
\mathcal{K}_{\mu \nu}^{\sigma}=\tilde{\Gamma}_{\mu \nu}^{\sigma} \quad \text { at } p
$$

Hence if torsion vanishes, the Levi-Civita connection coincides with the Weitzenböck connection, and both are flat. This can be done either for the basic metric $\gamma^{\mu \nu}$ or for the effective metric $G^{\mu \nu}$, but typically not for both simultaneously. In the present context, the Christoffel symbols will be $\mathfrak{h} \mathfrak{s - v a l u e d}$ in general, and so $\operatorname{are}^{17}$ the normal coordinates $y^{\mu}$.

In particular, consider harmonic coordinates local coordinates $y^{\mu}$ on $\mathcal{M}$ around $p$, which by definition satisfy $\square_{G} y^{\mu}=0$. We can demand in addition that at any given point they are also normal coordinates, so that

$$
\begin{aligned}
\square_{G} y^{\mu} & \equiv 0 \\
\left.\Gamma_{\nu \sigma}^{(G)}{ }_{\nu}^{\mu}\right|_{p} & =0
\end{aligned}
$$

To see that they exist, it suffices to note that one can find harmonic functions $y^{\mu}$ with any prescribed "boundary value" and normal derivative for any hypersurface through " $p$; thus one can choose the $\left.\Gamma_{\nu \sigma}^{(G)}{ }^{\mu}\right|_{p}$ freely up to $\Gamma_{(G)}^{\mu}$, which vanishes by the harmonic condition. We denote such coordinates as "harmonic normal coordinates", which will be used in the alternative derivation of the Ricci tensor in section A.5.

\subsection{Some useful identities}

It was shown in [8] that for symplectic manifolds, the Matrix or Poisson d'Alembertian $\square(2.20)$ is proportional to the metric d'Alembertian

$$
\square_{G} \phi=-\frac{1}{\sqrt{\left|G_{\mu \nu}\right|}} \partial_{\mu}\left(\sqrt{\left|G_{\mu \nu}\right|} G^{\mu \nu} \partial_{\nu} \phi\right)
$$

The same relation holds in the present reduced 4-dimensional setting: ${ }^{18}$

Lemma 5.1. The Matrix or Poisson d'Alembertian $\square(2.20)$ is related to the metric d'Alembertian $\square_{G} \phi$ via

$$
\square \phi=-\left\{Z_{\dot{\alpha}},\left\{Z^{\dot{\alpha}}, \phi\right\}\right\}=\rho^{2} \square_{G} \phi
$$

\footnotetext{
${ }^{17}$ Using $\mathfrak{h} \mathfrak{s}$-valued coordinates $y^{\mu}$ on $\mathcal{M}$ simply amounts to a deformation of the bundle projection $\Pi$ (2.22). The algebra generated by these 4 generators $y^{\mu}$ is formally isomorphic to the algebra of functions on $\mathcal{C}^{\infty}\left(\mathbb{R}^{3,1}\right)$.

${ }^{18}$ This is bound to hold due to the covariant form (4.9) of the kinetic term.
} 
in the asymptotic regime, ${ }^{19}$ with $\rho$ given in (4.11). Furthermore, the following identities hold

$$
\begin{aligned}
\Gamma_{\rho \mu}{ }^{\rho} & =\rho_{M}^{-1} \partial_{\mu} \rho_{M} \\
\square y^{\sigma} & =\partial_{\mu} \gamma^{\mu \sigma}+\Gamma_{\rho}^{\sigma \rho}=-\Gamma_{\mu}{ }^{\mu \sigma}=\Gamma_{\nu \rho}{ }^{\mu} \gamma^{\nu \rho}=\Gamma_{\nu \rho}^{(G)}{ }^{\mu} \gamma^{\nu \rho} \\
\Gamma_{\rho \mu}{ }^{\mu} & =-\partial_{\rho} \ln \left(\sqrt{\left|\gamma^{\mu \nu}\right|}\right) .
\end{aligned}
$$

This is proved in appendix A.3, by writing out $\square$ using vielbein and Weitzenböck connection and using (2.52). This is a generalization of a similar result in [8] for symplectic branes. It leads to the following explicit formulas for the contraction of the (con)torsion:

Lemma 5.2. The (con)torsion associated to the basic frame satisfies

$$
T_{\mu \sigma}{ }^{\mu}=K_{\mu \sigma}{ }^{\mu}=\frac{2}{\rho} \partial_{\sigma} \rho .
$$

For the rescaled frame resp. effective metric, we have

$$
\mathcal{T}_{\mu \sigma}{ }^{\mu}=\mathcal{K}_{\mu \sigma}{ }^{\mu}=-\rho^{-1} \partial_{\sigma} \rho .
$$

Proof. Using the above results, we can evaluate the contraction of the torsion

$$
\begin{aligned}
T_{\mu \rho}{ }^{\mu} & =\Gamma_{\mu \rho}{ }^{\mu}-\Gamma_{\rho \mu}{ }^{\mu}=\partial_{\rho}\left(\ln \left(\sqrt{\left|\gamma^{\mu \nu}\right|}\right)+\ln \rho_{M}\right) \\
& =\partial_{\rho} \ln \left(\rho_{M} \sqrt{\left|\gamma^{\mu \nu}\right|}\right)=\partial_{\rho} \ln \left(\rho^{2}\right)
\end{aligned}
$$

using $\sqrt{\mid \gamma^{\mu \nu \mid}}=\rho^{2} \rho_{M}^{-1}$, and

$$
K_{\mu \nu}^{\mu}=\frac{1}{2}\left(T_{\mu \nu}^{\mu}+T_{\mu}^{\mu}{ }_{\nu}-T_{\nu}^{\mu}{ }_{\mu}\right)=T_{\mu \nu}^{\mu}
$$

using (5.15). For the rescaled frame, (5.23) gives

$$
\tilde{\Gamma}_{\mu \sigma}^{\mu}=\Gamma_{\mu \sigma}^{\mu}+\rho^{-1} \partial_{\sigma} \rho, \quad \tilde{\Gamma}_{\nu \mu}^{\mu}=\Gamma_{\nu \mu}^{\mu}+\frac{4}{\rho} \partial_{\nu} \rho
$$

Therefore

$$
\mathcal{T}_{\mu \sigma}{ }^{\mu}=\tilde{\Gamma}_{\mu \sigma}{ }^{\mu}-\tilde{\Gamma}_{\sigma \mu}{ }^{\mu}=T_{\mu \sigma}{ }^{\mu}-\frac{3}{\rho} \partial_{\sigma} \rho=-\rho^{-1} \partial_{\sigma} \rho=\mathcal{K}_{\mu \sigma}{ }^{\mu} .
$$

\subsection{Equation of motion for the torsion}

Now consider the equation of motion for torsion, which results from the basic matrix equation of motion (2.3)

$$
\left\{Z^{\dot{\alpha}}, \hat{\Theta}_{\dot{\alpha} \dot{\beta}}\right\}=m^{2} Z_{\dot{\beta}}
$$

\footnotetext{
${ }^{19}$ The only "approximation" used is (2.52), which is established in the asymptotic regime only, although it appears to hold more generally.
} 
in vacuum. Recalling $T_{\dot{\alpha} \dot{\beta}}{ }^{\mu}=\left\{\hat{\Theta}_{\dot{\alpha} \dot{\beta}}, y^{\mu}\right\}$, we obtain the coordinate version from

$$
\begin{aligned}
m^{2}\left\{Z_{\dot{\beta}}, y^{\mu}\right\} & =\left\{\left\{Z^{\dot{\alpha}}, \hat{\Theta}_{\dot{\alpha} \dot{\beta}}\right\}, y^{\mu}\right\}=-\left\{\left\{\hat{\Theta}_{\dot{\alpha} \dot{\beta}}, y^{\mu}\right\}, Z^{\dot{\alpha}}\right\}-\left\{\left\{y^{\mu}, Z^{\dot{\alpha}}\right\}, \hat{\Theta}_{\dot{\alpha} \dot{\beta}}\right\} \\
& =\left\{Z^{\dot{\alpha}}, T_{\dot{\alpha} \dot{\beta}}{ }^{\mu}\right\}+\left\{E^{\dot{\alpha} \mu}, \hat{\Theta}_{\dot{\alpha} \dot{\beta}}\right\} .
\end{aligned}
$$

The second term can be rewritten as

$$
\left\{E^{\dot{\alpha} \mu}, \hat{\Theta}_{\dot{\alpha} \dot{\beta}}\right\} \sim-\partial_{\nu} E^{\dot{\alpha} \mu} T_{\dot{\alpha} \dot{\beta}}{ }^{\nu}=\Gamma_{\nu \sigma}{ }^{\mu} E^{\dot{\alpha} \sigma} T_{\dot{\alpha} \dot{\beta}}{ }^{\nu}=\left(\Gamma_{\sigma \nu}{ }^{\mu}+T_{\nu \sigma}{ }^{\mu}\right) T_{\dot{\beta}}^{\sigma}{ }^{\nu}
$$

using the approximation (2.49) in the asymptotic regime. Furthermore, we note that

$$
\begin{aligned}
\nabla_{\nu} T_{\dot{\beta}}^{\nu}{ }^{\mu} & =\nabla_{\nu}\left(E_{\dot{\alpha}}{ }^{\nu} T_{\dot{\beta}}^{\dot{\alpha}}{ }^{\mu}\right)=E_{\dot{\alpha}}{ }^{\nu} \nabla_{\nu} T_{\dot{\beta}}^{\dot{\alpha}}{ }^{\mu}=E_{\dot{\alpha}}{ }^{\nu}\left(\partial_{\nu} T_{\dot{\beta}}^{\dot{\alpha}}{ }^{\mu}+\Gamma_{\nu \sigma}{ }^{\mu} T_{\dot{\beta}}^{\dot{\alpha}}{ }^{\sigma}\right) \\
& =\left\{Z_{\dot{\alpha}}, T_{\dot{\beta}}^{\dot{\alpha}}{ }^{\mu}\right\}+\Gamma_{\sigma \nu}{ }^{\mu} T_{\dot{\beta}}^{\sigma}{ }^{\nu}
\end{aligned}
$$

since $\nabla E=0$. Combining this we obtain

$$
m^{2}\left\{Z_{\dot{\beta}}, y^{\mu}\right\}=\nabla_{\nu} T_{\dot{\beta}}^{\nu}{ }^{\mu}+T_{\nu \sigma}{ }^{\mu} T_{\dot{\beta}}^{\sigma}{ }^{\nu}
$$

which amounts to

$$
\nabla_{\nu} T_{\rho}^{\nu}{ }^{\mu}+T_{\nu \sigma}^{\mu} T_{\rho}^{\sigma}{ }_{\rho}^{\nu}=m^{2} \delta_{\rho}^{\mu} .
$$

Upon lowering an index with $\gamma_{\mu \nu}$ we obtain

$$
\nabla_{\nu} T_{\rho \mu}^{\nu}+T_{\nu}{ }^{\sigma} T_{\sigma \rho}{ }^{\nu}=m^{2} \gamma_{\rho \mu} .
$$

This non-linear equation encodes the non-linear structure of the Yang-Mills equations of motion (5.41). Contracting with $\gamma^{\mu \rho}$ gives

$$
\nabla_{\nu} T_{\mu}^{\nu}{ }^{\mu}+T_{\nu}{ }_{\mu}{ }_{\mu} T_{\sigma \rho}{ }^{\nu} \gamma^{\mu \rho}=4 m^{2} .
$$

The first term can be evaluated using (5.35) and (5.31) and (5.33) as

$$
\begin{aligned}
\nabla_{\nu} T_{\mu}^{\nu}{ }^{\mu} & =-2 \nabla_{\nu}\left(\rho^{-1} \gamma^{\nu \sigma} \partial_{\sigma} \rho\right)=2 \rho^{-2} \partial_{\nu} \rho \gamma^{\nu \sigma} \partial_{\sigma} \rho-2 \rho^{-1} \gamma^{\nu \sigma} \nabla_{\nu} \partial_{\sigma} \rho \\
& =2 \rho^{-2} \partial_{\nu} \rho \gamma^{\nu \sigma} \partial_{\sigma} \rho-2 \rho^{-1} \gamma^{\nu \sigma}\left(\partial_{\nu} \partial_{\sigma} \rho-\Gamma_{\nu \sigma}{ }^{\mu} \partial_{\mu} \rho\right) \\
& =2 G^{\nu \sigma} \partial_{\nu} \rho \partial_{\sigma} \rho-2 \rho G^{\nu \sigma}\left(\partial_{\nu} \partial_{\sigma} \rho-\Gamma^{(G)}{ }_{\nu \sigma}^{\mu} \partial_{\mu} \rho\right) \\
& =2\left(G^{\mu \nu} \partial_{\mu} \rho \partial_{\nu} \rho+\rho \square_{G} \rho\right) .
\end{aligned}
$$

We therefore obtain

$$
\rho \square_{G} \rho+G^{\mu \nu} \partial_{\mu} \rho \partial_{\nu} \rho=2 m^{2}-\frac{1}{2} T_{\nu}{ }^{\sigma}{ }_{\mu} T_{\sigma \rho}{ }^{\nu} \gamma^{\mu \rho} .
$$

According to the discussion in section 2.3, all these equations are exact for $\mathcal{C}^{0}$, and asymptotic for the higher spin components. We verify in appendix A.4 that these equations are indeed satisfied exactly for the background solution and its torsion. If desired, (5.47) can be rewritten in terms of the Levi-Civita connection using (5.20),

$$
m^{2} \gamma_{\rho \mu}=\nabla_{\nu}^{(G)} T_{\rho \mu}^{\nu}+\mathcal{K}_{\nu \rho}{ }^{\sigma} T^{\nu}{ }_{\sigma \mu}+\mathcal{K}_{\nu \mu}{ }^{\sigma} T_{\rho \sigma}^{\nu}+T_{\nu}{ }^{\sigma}{ }_{\mu} T_{\sigma \rho}{ }^{\nu} .
$$

It could be expressed in terms of the torsion only, but this does not lead to a simpler expression. Together with the equation (5.72) for the Ricci tensor and the Bianchi identity below, this provides a closed system of equations for the metric and the torsion. 


\subsection{Bianchi identity}

There is a Bianchi-type identity for the torsion, which results from the Jacobi identity

$$
\left\{Z_{\dot{\gamma}}, \hat{\Theta}_{\dot{\alpha} \dot{\beta}}\right\}+(\operatorname{cycl})=0
$$

where (cycl) indicates cyclic permutations in $\dot{\alpha}, \dot{\beta}, \dot{\gamma}$. Its coordinate version is

$$
\begin{aligned}
0 & =\left\{\left\{Z_{\dot{\gamma}}, \hat{\Theta}_{\dot{\alpha} \dot{\beta}}\right\}, y^{\mu}\right\}+(\mathrm{cycl}) \\
& =-\left\{\left\{\hat{\Theta}_{\dot{\alpha} \dot{\beta}}, y^{\mu}\right\}, Z_{\dot{\gamma}}\right\}-\left\{\left\{y^{\mu}, Z_{\dot{\gamma}}\right\}, \hat{\Theta}_{\dot{\alpha} \dot{\beta}}\right\}+(\mathrm{cycl}) \\
& =\left\{Z_{\dot{\gamma}}, T_{\dot{\alpha} \dot{\beta}}{ }^{\mu}\right\}+\left\{E_{\dot{\gamma}}{ }^{\mu}, \hat{\Theta}_{\dot{\alpha} \dot{\beta}}\right\}+(\mathrm{cycl}) \\
& \sim \nabla_{\dot{\gamma}} T_{\dot{\alpha} \dot{\beta}}{ }^{\mu}-\Gamma_{\dot{\gamma} \sigma}{ }^{\mu} T_{\dot{\alpha} \dot{\beta}}{ }^{\sigma}-T_{\dot{\alpha} \dot{\beta}}{ }^{\nu} \partial_{\nu} E_{\dot{\gamma}}{ }^{\mu}+(\mathrm{cycl}) \\
& =\nabla_{\dot{\gamma}} T_{\dot{\alpha} \dot{\beta}}{ }^{\mu}-T_{\dot{\alpha} \dot{\beta}}{ }^{\sigma} \Gamma_{\dot{\gamma} \sigma}{ }^{\mu}+T_{\dot{\alpha} \dot{\beta}}{ }^{\nu} \Gamma_{\nu \dot{\gamma}}{ }^{\mu}+(\mathrm{cycl}) \\
& =\nabla_{\dot{\gamma}} T_{\dot{\alpha} \dot{\beta}}{ }^{\mu}+T_{\dot{\alpha} \dot{\beta}}{ }^{\nu} T_{\nu \dot{\gamma}}{ }^{\mu}+(\mathrm{cycl})
\end{aligned}
$$

using again (2.49), or equivalently

$$
0=\nabla_{\sigma} T_{\lambda \rho}^{\mu}+\nabla_{\lambda} T_{\rho \sigma}^{\mu}+\nabla_{\rho} T_{\sigma \lambda}^{\mu}+T_{\lambda \rho}{ }^{\nu} T_{\nu \sigma}{ }^{\mu}+T_{\rho \sigma}{ }^{\nu} T_{\nu \lambda}{ }^{\mu}+T_{\sigma \lambda}{ }^{\nu} T_{\nu \rho}^{\mu}
$$

which is cyclic in $\lambda, \rho, \sigma$. Together with (5.47) we obtained an analog of the Yang-Mills equations. Contracting $\sigma \mu$, this gives

$$
0=\nabla_{\sigma} T_{\lambda \rho}^{\sigma}+\nabla_{\lambda} T_{\rho \sigma}^{\sigma}-\nabla_{\rho} T_{\lambda \sigma}^{\sigma}-2 \rho^{-1} \partial_{\nu} \rho T_{\lambda \rho}^{\nu}
$$

using Lemma 5.2. The middle terms can be rewritten using

$$
\left(\nabla_{\mu} \partial_{\nu}-\nabla_{\nu} \partial_{\mu}\right) \rho=-\Gamma_{\mu \nu}^{\sigma} \partial_{\sigma} \phi+\Gamma_{\nu \mu}^{\sigma} \partial_{\sigma} \rho=-T_{\mu \nu}^{\sigma} \partial_{\sigma} \rho
$$

and we obtain the identity

$$
\nabla_{\sigma} T_{\lambda \rho}^{\sigma}=0 .
$$

Contracting (5.54) with $\gamma^{\sigma \lambda}$ does not give any non-trivial relation.

\subsection{Vacuum equation for the Ricci tensor}

Now we compute the Ricci tensor for the Levi-Civita connection associated with the effective metric $G^{\mu \nu}$. This is achieved by expressing the Riemann tensor in terms of the torsion. We start from

$$
\begin{aligned}
\mathcal{R}_{\mu \nu}{ }^{\lambda} \sigma & =\partial_{\mu} \Gamma^{(G)}{ }_{\nu \sigma}^{\lambda}-\partial_{\nu} \Gamma^{(G)}{ }_{\mu \sigma}{ }^{\lambda}+\Gamma^{(G)}{ }_{\mu \rho}^{\lambda} \Gamma^{(G)}{ }_{\nu \sigma}{ }^{\rho}-\Gamma^{(G)}{ }_{\nu \rho}^{\lambda} \Gamma^{(G)}{ }_{\mu \sigma}{ }^{\rho} \\
\mathcal{R}_{\nu \sigma} & =\partial_{\mu} \Gamma^{(G)}{ }_{\nu \sigma}{ }^{\mu}-\partial_{\nu} \Gamma^{(G)}{ }_{\mu \sigma}{ }^{\mu}+\Gamma^{(G)}{ }_{\mu \rho}^{\mu} \Gamma^{(G)}{ }_{\nu \sigma}{ }^{\rho}-\Gamma^{(G)}{ }_{\nu \rho}^{\mu} \Gamma^{(G)}{ }_{\mu \sigma}{ }^{\rho} .
\end{aligned}
$$

In (Riemann) normal coordinates at $p \in \mathcal{M}$, this simplifies using (5.18) as

$$
\begin{aligned}
\mathcal{R}_{\mu \nu \sigma}{ }^{\lambda} & =\partial_{\mu}\left(\tilde{\Gamma}_{\nu \sigma}{ }^{\lambda}-\mathcal{K}_{\nu \sigma}{ }^{\lambda}\right)-\partial_{\nu}\left(\tilde{\Gamma}_{\mu \sigma}{ }^{\lambda}-\mathcal{K}_{\mu \sigma}{ }^{\lambda}\right) \\
\mathcal{R}_{\nu \sigma} & =\partial_{\mu}\left(\tilde{\Gamma}_{\nu \sigma}{ }^{\mu}-\mathcal{K}_{\nu \sigma}{ }^{\mu}\right)-\partial_{\nu}\left(\tilde{\Gamma}_{\mu \sigma}{ }^{\mu}-\mathcal{K}_{\mu \sigma}{ }^{\mu}\right) .
\end{aligned}
$$


Now we use the fact that the curvature of the Weitzenböck connection vanishes,

$$
\begin{aligned}
& 0=\partial_{\mu} \tilde{\Gamma}_{\nu \sigma}^{\lambda}-\partial_{\nu} \tilde{\Gamma}_{\mu \sigma}^{\lambda}+\tilde{\Gamma}_{\mu \rho}{ }^{\lambda} \tilde{\Gamma}_{\nu \sigma}{ }^{\rho}-\tilde{\Gamma}_{\nu \rho}{ }^{\lambda} \tilde{\Gamma}_{\mu \sigma}{ }^{\rho} \\
& 0=\partial_{\mu} \tilde{\Gamma}_{\nu \sigma}{ }^{\mu}-\partial_{\nu} \tilde{\Gamma}_{\mu \sigma}{ }^{\mu}+\tilde{\Gamma}_{\mu \rho}{ }^{\mu} \tilde{\Gamma}_{\nu \sigma}{ }^{\rho}-\tilde{\Gamma}_{\nu \rho}{ }^{\mu} \tilde{\Gamma}_{\mu \sigma}{ }^{\rho}
\end{aligned}
$$

and obtain the tensorial equations

$$
\begin{aligned}
\mathcal{R}_{\mu \nu \sigma}{ }^{\lambda} & =-\nabla_{\mu}^{(G)} \mathcal{K}_{\nu \sigma}{ }^{\lambda}+\nabla_{\nu}^{(G)} \mathcal{K}_{\mu \sigma}{ }^{\lambda}-\mathcal{K}_{\mu \rho}{ }^{\lambda} \mathcal{K}_{\nu \sigma}{ }^{\rho}+\mathcal{K}_{\nu \rho}{ }^{\lambda} \mathcal{K}_{\mu \sigma}{ }^{\rho} \\
\mathcal{R}_{\nu \sigma} & =-\nabla_{\mu}^{(G)} \mathcal{K}_{\nu \sigma}{ }^{\mu}+\nabla_{\nu}^{(G)} \mathcal{K}_{\mu \sigma}{ }^{\mu}-\mathcal{K}_{\mu \rho}{ }^{\mu} \mathcal{K}_{\nu \sigma}{ }^{\rho}+\mathcal{K}_{\nu \rho}{ }^{\mu} \mathcal{K}_{\mu \sigma}{ }^{\rho}
\end{aligned}
$$

using (5.28). It remains to evaluate the derivative terms of the contorsion. The first term can be evaluated using (5.19), which gives

$$
\nabla_{\mu}^{(G)} \mathcal{K}_{\nu \sigma}{ }^{\mu}=\nabla_{\mu}^{(G)} K_{\nu \sigma}{ }^{\mu}-G_{\nu \sigma}\left(\rho^{-1} \square_{G} \rho+\rho^{-2} \partial \rho \cdot \partial \rho\right)-\rho^{-1} \nabla_{\nu}^{(G)} \partial_{\sigma} \rho+\rho^{-2} \partial_{\nu} \rho \partial_{\sigma} \rho
$$

where

$$
\partial \rho \cdot \partial \rho:=G^{\mu \sigma} \partial_{\mu} \rho \partial_{\sigma} \rho
$$

Further, (5.36) gives

$$
\nabla_{\nu}^{(G)} \mathcal{K}_{\mu \sigma}{ }^{\mu}=-\nabla_{\nu}^{(G)}\left(\rho^{-1} \partial_{\sigma} \rho\right)=\rho^{-2} \partial_{\nu} \rho \partial_{\sigma} \rho-\rho^{-1} \nabla_{\nu}^{(G)} \partial_{\sigma} \rho
$$

so that

$$
-\nabla_{\mu}^{(G)} \mathcal{K}_{\nu \sigma}{ }^{\mu}+\nabla_{\nu}^{(G)} \mathcal{K}_{\mu \sigma}{ }^{\mu}=-\nabla_{\mu}^{(G)} K_{\nu \sigma}{ }^{\mu}+G_{\nu \sigma}\left(\rho^{-1} \square_{G} \rho+\rho^{-2} \partial \rho \cdot \partial \rho\right) .
$$

The first term can be evaluated as

$$
\nabla_{\mu}^{(G)} K_{\nu \sigma}{ }^{\mu}=\nabla_{\mu} K_{\nu \sigma}{ }^{\mu}+\mathcal{K}_{\mu \nu}{ }^{\rho} K_{\rho \sigma}{ }^{\mu}+\mathcal{K}_{\mu \sigma}{ }^{\rho} K_{\nu \rho}{ }^{\mu}
$$

using (5.20), (5.19) and (5.36). This gives after some straightforward algebra using (5.26)

$$
\mathcal{R}_{\nu \sigma}=-\nabla_{\mu} K_{\nu \sigma}^{\mu}-K_{\mu \nu}^{\rho} K_{\rho \sigma}^{\mu}+2 \rho^{-2} \partial_{\sigma} \rho \partial_{\nu} \rho+G_{\nu \sigma}\left(\rho^{-1} \square_{G} \rho+\rho^{-2} \partial \rho \cdot \partial \rho\right) .
$$

So far this is an identity. We now replace the contorsion with the torsion using (5.15) and use the Bianchi identity (5.57) as well as the equation of motion (5.47) for the torsion. This gives

$$
\begin{aligned}
\nabla_{\mu} K_{\nu \sigma}{ }^{\mu} & =\frac{1}{2} \nabla_{\mu}\left(T_{\nu \sigma}{ }^{\mu}+T_{\nu \sigma}^{\mu}-T_{\sigma}{ }_{\nu}{ }^{\prime}\right)=\frac{1}{2} \nabla_{\mu}\left(T_{\nu \sigma}^{\mu}+T_{\sigma \nu}^{\mu}\right) \\
& =-\frac{1}{2}\left(T_{\sigma}^{\rho \eta} T_{\eta \nu \rho}+T_{\nu}^{\rho \eta} T_{\eta \sigma \rho}\right)+m^{2} \gamma_{\nu \sigma},
\end{aligned}
$$

and we obtain the desired equation for the Ricci tensor of the effective metric in vacuum

$$
\begin{aligned}
\mathcal{R}_{\nu \sigma}[G]= & -\frac{1}{2}\left(T_{\rho}{ }^{\mu}{ }_{\sigma} T_{\nu \mu}{ }^{\rho}+T_{\rho}{ }^{\mu}{ }_{\nu} T_{\sigma \mu}{ }^{\rho}\right)-K_{\mu}{ }^{\rho}{ }_{\nu} K_{\rho}{ }^{\mu}{ }_{\sigma}+2 \rho^{-2} \partial_{\nu} \rho \partial_{\sigma} \rho \\
& +G_{\nu \sigma}\left(-\rho^{-2} m^{2}+\rho^{-1} \square_{G} \rho+G^{\mu \nu} \rho^{-2} \partial_{\mu} \rho \partial_{\nu} \rho\right) .
\end{aligned}
$$


This agrees precisely with the result (A.51) obtained in a more pedestrian way. The last term can be rewritten in terms of the torsion using the contracted equations of motion (5.50),

$$
\begin{aligned}
\mathcal{R}_{\nu \sigma}= & -\frac{1}{2}\left(T_{\rho}{ }^{\mu}{ }_{\sigma} T_{\nu \mu}{ }^{\rho}+T_{\rho}{ }_{\nu}{ }_{\nu} T_{\sigma \mu}{ }^{\rho}\right)-K_{\mu}{ }^{\rho}{ }_{\nu} K_{\rho}{ }^{\mu}{ }_{\sigma}+2 \rho^{-2} \partial_{\nu} \rho \partial_{\sigma} \rho \\
& +G_{\nu \sigma}\left(\rho^{-2} m^{2}-\frac{1}{2} T_{\nu}{ }^{\sigma}{ }_{\mu} T_{\sigma \rho}{ }^{\nu} G^{\mu \rho}\right) .
\end{aligned}
$$

This is an algebraic equation for the Ricci tensor in terms of torsion. ${ }^{20}$ The Ricci scalar is obtained by contracting with $G^{\nu \sigma}$,

$$
\begin{aligned}
\mathcal{R}[G] & =-T_{\rho}{ }^{\mu \nu} T_{\mu \nu}{ }^{\rho}-K_{\mu \nu}{ }^{\rho} K_{\rho}{ }^{\mu \nu}+2 \rho^{-2} \partial \rho \cdot \partial \rho+4 \rho^{-2} m^{2} \\
& =-\frac{1}{2} T^{\sigma \mu \rho} T_{\mu \rho \sigma}-\frac{1}{4} T^{\mu \sigma \rho} T_{\mu \sigma \rho}+2 \rho^{-2}\left(\partial \rho \cdot \partial \rho+2 m^{2}\right)
\end{aligned}
$$

using (A.56) in the last step. Hence the present vacuum equations can be written as Einstein equations in the form

$$
\mathcal{G}_{\mu \nu}=\mathcal{R}_{\mu \nu}-\frac{1}{2} G_{\mu \nu} \mathcal{R}=8 \pi \mathbf{T}_{\mu \nu}
$$

with an effective energy-momentum tensor due to the torsion,

$$
\begin{aligned}
8 \pi \mathbf{T}_{\mu \nu}= & -\frac{1}{2}\left(T_{\rho}^{\delta}{ }_{\nu} T_{\mu \delta}{ }^{\rho}+T_{\rho}^{\delta}{ }_{\mu} T_{\nu \delta}{ }^{\rho}\right)-K_{\delta}{ }_{\mu}{ }_{\mu} K_{\rho}{ }^{\delta}{ }_{\nu}+2 \rho^{-2} \partial_{\mu} \rho \partial_{\nu} \rho \\
& +G_{\mu \nu}\left(-\frac{1}{4} T^{\sigma \delta \rho} T_{\delta \rho \sigma}+\frac{1}{8} T^{\delta \sigma \rho} T_{\delta \sigma \rho}-\rho^{-2} \partial \rho \cdot \partial \rho-3 R^{-2} \rho^{-2}\right)
\end{aligned}
$$

recalling that $m^{2}=3 R^{-2}$. This is verified for the cosmic background in section (A.4). The conservation law $\nabla_{(G)}^{\nu} \mathbf{T}_{\nu \mu}=0$ is guaranteed at least in vacuum because the r.h.s. simply computes the Einstein tensor, which is conserved.

The above equations (5.47), (5.54) and (5.72) for the torsion and Ricci tensor provide a closed system of non-linear equations which govern the emergent gravity on the present

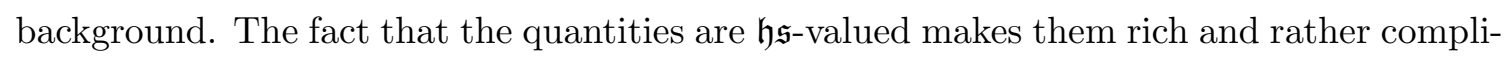
cated. ${ }^{21}$ Although the present derivation is restricted to the asymptotic regime for the $\mathfrak{h} \mathfrak{s}$ sector, the equations are exact for the lowest components in $\mathcal{C}^{0}$, as verified for the cosmological background. Using covariance under higher-spin gauge transformations, it should be possible to largely transform away the $\mathfrak{h} \mathfrak{s}$-components in many situations, and the exact equations for the $\mathcal{C}^{0}$ component should be accessible to analytic investigation.

\subsection{Discussion and further considerations}

The crucial point of the above result is that $\mathbf{T}_{\mu \nu}=O(T T)$ is quadratic in the torsion, as appropriate for an energy-momentum tensor. In contrast, the Riemann tensor (5.61) contains a (derivative) term which is linear in the torsion, hence any non-trivial geometry

\footnotetext{
${ }^{20}$ We can consider $\rho^{-1} \partial_{\nu} \rho=\mathcal{K}_{\mu}{ }^{\mu}{ }_{\nu}$ as part of the torsion (5.36).

${ }^{21}$ Recall that $\mathfrak{h} \mathfrak{s}$ is a commutative in the semi-classical limit here, hence there is no ordering ambiguity.
} 
has non-trivial torsion. However, this linear contribution vanishes on-shell for the Ricci tensor, so that vacuum geometries are Ricci-flat up to higher-order (non-linear) contributions. This means that the present theory is a serious candidate for gravity, and deviations from GR (at least in vacuum) are restricted to the non-linear regime.

It remains to quantify the deviations of the present theory from GR. One regime where deviations will surely arise is the strong gravity regime, where the Riemann tensor is large in some sense. That is the regime where deviations from GR typically arise in alternative approaches to gravity such as string theory. However there is another regime where torsion may lead to significant modifications here, namely for very large, massive objects as discussed in the next section. This new mechanism arises from the self-coupling of torsion due to (5.47), and it might mimic the presence of dark matter as discussed below.

Torsion as a "dark matter". Consider first the linearization of the torsion

$$
T_{\rho \nu}^{\mu}=\bar{T}_{\rho \nu}{ }^{\mu}+t_{\rho \nu}{ }^{\mu}
$$

around the cosmic background torsion $\bar{T}=O\left(\frac{1}{a(t)}\right)$ (A.26). Since the contributions from the background cancel, the eom (5.51) takes the following schematic form

$$
-\nabla_{\nu}^{(G)} t_{\rho \mu}^{\nu} \sim-\frac{3}{a(t)^{2}} \delta G_{\rho \mu}+\frac{1}{a(t)}(t)_{\rho \mu}+(t t)_{\rho \mu} .
$$

The constant and linear terms on the r.h.s. are suppressed by the cosmic scale factor $\frac{1}{a(t)}$. However, since the torsion is smaller than the cosmic background in the linearized regime, it will not be very significant physically. Therefore we need to consider the non-linear regime,

$$
\nabla_{\nu}^{(G)} t_{\rho \mu}^{\nu} \sim-(t t)_{\rho \mu}
$$

To get a rough qualitative idea what this could mean, consider the simplified radial equation

$$
t^{\prime}=-t^{2}
$$

for $t=t(r)$, where $r$ is the distance to the center of some massive object. This should give a reasonable picture for the radial dependence of the torsion tensor. That equation has the general solution

$$
t(r)=\frac{1}{r+c}
$$

for some constant $c$. This leads to an effective energy-momentum tensor $8 \pi \mathbf{T}_{\mu \nu} \sim \frac{1}{(r+c)^{2}}(5.73)$ as a source of the Einstein tensor, which would behave like a dark matter halo with density profile $\rho_{\mathrm{DM}}(r) \sim \frac{1}{(r+c)^{2}}$ corresponding to a total mass

$$
M_{D M}(r) \sim c+r+O\left(\frac{1}{r}\right), \quad v_{\text {rot }}(r) \sim \text { const }
$$

leading to a rotation velocity $v_{\text {rot }}(r)$ which is roughly independent of the distance $r$ to the (galactic) center. This is indeed what is typically observed. The scale parameter $c$ should be determined by continuity in a refined treatment, and it is presumably set by the "size" 
or mass of the object. At very large distances, the torsion (5.78) will merge to that of the cosmic background, leading to a natural cutoff for the effect. For small masses or objects, there will not be sufficient space for (5.78) to rise significantly above the background, so that the effect should be significant only for very large objects such as galaxies.

Needless to say that this crude qualitative consideration needs to be considerably refined before quantitative statements can be made, and the coupling to matter needs to be understood and taken into account properly. In any case, it is intriguing to obtain a qualitatively reasonable first estimate, and it is also encouraging that the present mechanism based on dynamical torsion is sufficiently rich that different types of behavior might be produced.

Coupling to matter. This paper is restricted to the vacuum geometry of the model. To properly talk about gravity we should of course take matter into account, which is indeed an intrinsic part of the IKKT matrix model. It is clear that the kinematics of matter is properly governed by the metric; this is how the metric was identified. ${ }^{22}$ However, the non-trivial question is how matter acts as a source for torsion and the Ricci tensor. It is not clear what is the best way to work this out, and we postpone this question to future work. However, a few comments can be made at this point: first of all, due to the (higher-spin) covariance of the theory it is highly plausible that the energy-momentum tensor for matter will arise on the r.h.s. of the Einstein equations. However there will also be higher-derivative terms, and the question is if the standard contribution dominates the higher-derivative contributions; see also the related discussion in [9]. Covariance will strongly restrict the possible terms, and it is certainly plausible that the energy-momentum tensor will dominate. Due to the presence of several scales on the background this must be studied in detail, in order to identify the effective Newton constant. Since quantum effects are typically significant in this context, this may not be a trivial task.

Similarly, the effect of matter on torsion must be understood. This is expected to be small since for bosonic matter there should not source torsion at all (as only the metric appears in the kinetic term), and for fermions the effect is expected to be small as well, due to the supersymmetry of the underlying matrix model.

\section{Conclusion and outlook}

The present paper provides a tensorial description of the vacuum sector of the effective gravity which arises on a solution of the IKKT matrix models found in [9], interpreted as FLRW space-time. The noncommutative Yang-Mills gauge theory is cast into a geometric form which makes the gauge invariance manifest, in terms of a higher-spin generalization of volume-preserving diffeomorphisms. The crucial concept turns out to be torsion, or rather a higher-spin generalization of torsion, which encodes the quantum structure of space-time and provides its semi-classical shadow.

Torsion turns out to be an independent and additional physical quantity besides the metric, and the Einstein equations for vacuum are modified through an effective energy-

\footnotetext{
${ }^{22}$ As discussed in [8], the Dirac operator for fermions in the IKKT model is also based on the effective frame as it should. This should be elaborated in more detail elsewhere.
} 
momentum tensor due to torsion. A non-linear equation for torsion is obtained, which encodes the underlying Yang-Mills-type equations of motion of the matrix model. This equation is exact for the standard (lowest-spin) tensorial components, but obtained only in an intermediate ("asymptotic") regime for the higher-spin components.

Moreover, we have argued that at least in the vacuum sector, the modification of GR due to torsion should be small except for very large objects such as galaxies, and on cosmic scales. The point is that torsion enters quadratically in the effective energy-momentum tensor, while it is governed itself by some non-linear PDE. A rough qualitative estimate suggests that it could indeed behave like an apparent dark matter halo around galaxies. In principle, the equations obtained in this paper should allow to obtain a quantitative description for this effect which can be tested.

The theory of gravity obtained in this way is governed by an action which is very different from the Einstein-Hilbert action. Unlike in the teleparallel formulation of general relativity, there is no way to rewrite the matrix model as local action in terms of the torsion, metric and frame. It is precisely this non-standard non-geometric origin which makes the present approach to gravity so interesting and potentially far-reaching.

As in all higher-spin theories, an important question is if the theory reduces in some suitable regime to an ordinary (modified) gravity theory with spin $\leq 2$. The presence of both an IR scale (given by the cosmic curvature scale) and a UV scale (given by the scale of noncommutativity (2.30)) leads to the hope that this may be the case in the present framework. However, this needs to be studied in future work.

Moreover, the present paper is limited to the vacuum sector of gravity. This restriction is only due to technical reasons, and obviously needs to be removed in future work. In principle, everything should follow from the underlying matrix model, and matter will certainly influence the geometry in some way consistent with the (generalized) covariance. Moreover, the framework of matrix models allows to make sense of the path integral. In particular for the maximally supersymmetric IKKT model, one may reasonably hope that the present (semi-) classical treatment is not too far from the full quantum theory. The extra structure required for an interesting matter sector can naturally arise from fuzzy extra dimensions realized by the extra 6 bosonic matrices in the model, as discussed e.g. in [35-38], see also [39].

Finally, a general message is that we ought to be cautious in extrapolating general relativity to regimes where it was not directly tested. The present theory may reproduce GR quite well in intermediate regimes and it has a healthy linear excitation spectrum without ghosts [10], but it certainly differs significantly on very long scales. Thus the correct theory of gravity may be far richer than GR, and the puzzles of dark matter, dark energy and the cosmological constant may well be evidence supporting such a picture.

\section{Acknowledgments}

I would like to thank Loriano Bonora and Stefan Fredenhagen for useful discussions, and Marcus Sperling for collaboration in the early stages of this project. This work was supported by the Austrian Science Fund (FWF) grant P32086. 


\section{A Technical supplements}

\section{A.1 Volume-preserving diffeomorphisms}

Consider the vector field (3.3) associated a gauge transformation generated by $\Lambda^{(s)} \in \mathcal{C}^{s}$

$$
\xi^{\mu}:=\left\{\Lambda^{(s)}, x^{\mu}\right\}=\xi_{+}^{\mu}+\xi_{-}^{\mu} \quad \in \mathcal{C}^{s+1} \oplus \mathcal{C}^{s-1}
$$

Using the basic identities (A.35) in [9], it is easy to see that these components satisfy the following constraint

$$
\left\{t_{\mu}, \xi_{+}^{\mu}\right\}=-\frac{s+3}{R^{2} \sinh (\eta)} x_{\mu} \xi_{+}^{\mu}, \quad\left\{t_{\mu}, \xi_{-}^{\mu}\right\}=-\frac{-s+2}{R^{2} \sinh (\eta)} x_{\mu} \xi_{-}^{\mu}
$$

or equivalently

$$
\bar{\nabla}_{\mu}\left(\beta^{s+3} \xi_{+}^{\mu}\right)=0=\bar{\nabla}_{\mu}\left(\beta^{-s+2} \xi_{-}^{\mu}\right), \quad \beta=\frac{1}{\sinh (\eta)}
$$

Here $\bar{\nabla}$ is the Levi-Civita derivative w.r.t. the cosmic background metric [12]. In this sense, $\xi^{\mu}$ can be interpreted as volume-preserving higher spin diffeomorphism.

\section{A.2 Calculations for the Poisson bracket}

We claim that the following formula realizes the ansatz (2.36) for the Poisson brackets:

$$
\begin{aligned}
\cosh ^{2}(\eta)\{f, g\}= & \left(\sinh (\eta)\left\{t_{\mu}, f\right\}-\frac{1}{r^{2} R^{2}}\left\{f, x_{\nu}\right\}\left(\theta^{\nu \mu}+r^{2} \sinh ^{-1}(\eta)\left(t^{\nu} x^{\mu}-x^{\nu} t^{\mu}\right)\right)\right)\left\{x_{\mu}, g\right\} \\
& +\left(\sinh (\eta)\left\{f, x^{\mu}\right\}+\left\{f, t_{\nu}\right\} \theta^{\nu \mu}\right)\left\{t_{\mu}, g\right\}
\end{aligned}
$$

The two terms look different due to the ambiguity in (2.36), and different forms can be obtained using

$$
\begin{aligned}
\left\{f, t_{\nu}\right\}\left(t^{\nu} x^{\mu}-x^{\nu} t^{\mu}\right)\left\{t_{\mu}, g\right\} & =\left\{f, t_{\nu}\right\} t^{\nu} x^{\mu}\left\{t_{\mu}, g\right\}-\left\{f, t_{\nu}\right\} x^{\nu} t^{\mu}\left\{t_{\mu}, g\right\} \\
& =\frac{1}{r^{2} R^{2}}\left\{f, x_{\nu}\right\}\left(x^{\nu} t^{\mu}-t^{\nu} x^{\mu}\right)\left\{x_{\mu}, g\right\}
\end{aligned}
$$

This leads to the following closed formulas for the derivatives $\partial$ and $\mathcal{D}$ in (2.39):

$$
\begin{aligned}
\cosh ^{2}(\eta) \oiint_{\mu} f & =\sinh (\eta)\left\{t_{\mu}, f\right\}-\frac{1}{r^{2} R^{2}}\left(\theta^{\nu \mu}+r^{2} \sinh ^{-1}(\eta)\left(t^{\nu} x^{\mu}-x^{\nu} t^{\mu}\right)\right)\left\{f, x_{\nu}\right\} \\
\cosh ^{2}(\eta) \mathcal{D}^{\mu}(f) & =\sinh (\eta)\left\{f, x^{\mu}\right\}+\theta^{\nu \mu}\left\{f, t_{\nu}\right\} .
\end{aligned}
$$


For the generators, this gives

$$
\begin{aligned}
\cosh ^{2}(\eta) \mathcal{D}^{\mu} t_{\rho} & =\sinh (\eta)\left\{t_{\rho}, x^{\mu}\right\}+\left\{t_{\rho}, t_{\nu}\right\} \theta^{\nu \mu} \\
& =\sinh ^{2}(\eta) \delta_{\rho}^{\mu}-\frac{1}{r^{2} R^{2}} \theta^{\rho \nu} \theta^{\nu \mu} \\
& =\cosh ^{2}(\eta) P_{\perp}^{\rho \mu}-r^{2} t^{\rho} t^{\mu}, \quad P_{\perp}^{\rho \mu}=\eta^{\rho \mu}-\frac{1}{x_{\nu} x^{\nu}} x^{\rho} x^{\mu} \\
\cosh ^{2}(\eta) \mathcal{D}^{\mu} x_{\rho} & =\sinh (\eta) \theta^{\rho \mu}-\sinh (\eta) \theta^{\rho \mu}=0 \\
\cosh ^{2}(\eta) \partial^{\mu} x_{\rho} & =\sinh (\eta)\left\{t_{\mu}, x_{\rho}\right\}-\frac{1}{r^{2} R^{2}}\left\{x_{\rho}, x_{\nu}\right\}\left(\theta^{\nu \mu}+r^{2} \sinh ^{-1}(\eta)\left(t^{\nu} x^{\mu}-x^{\nu} t^{\mu}\right)\right) \\
& =\sinh ^{2}(\eta) \delta_{\mu}^{\rho}-\frac{1}{r^{2} R^{2}}\left(\theta^{\rho \nu} \theta^{\nu \mu}+r^{2} \sinh ^{-1}(\eta)\left(\theta^{\rho \nu} t^{\nu} x^{\mu}-\theta^{\rho \nu} x^{\nu} t^{\mu}\right)\right) \\
& =\cosh ^{2}(\eta) \eta^{\rho \nu} \\
\cosh ^{2}(\eta) \partial^{\mu} t_{\rho} & =\sinh (\eta)\left\{t_{\mu}, t_{\rho}\right\}-\frac{1}{r^{2} R^{2}}\left\{t_{\rho}, x_{\nu}\right\}\left(\theta^{\nu \mu}+r^{2} \sinh ^{-1}(\eta)\left(t^{\nu} x^{\mu}-x^{\nu} t^{\mu}\right)\right) \\
& =-\frac{1}{r^{2} R^{2}} \sinh (\eta) \theta^{\mu \rho}-\frac{1}{r^{2} R^{2}} \sinh (\eta)\left(\theta^{\rho \mu}+r^{2} \sinh ^{-1}(\eta)\left(t^{\rho} x^{\mu}-x^{\rho} t^{\mu}\right)\right) \\
& =-\frac{1}{R^{2}}\left(t^{\rho} x^{\mu}-x^{\rho} t^{\mu}\right)
\end{aligned}
$$

One can then verify (A.4) and (2.38) explicitly.

Locally rescaled generators. To get a better intuition for the Poisson brackets, let us define adapted momentum generators for some cosmic time scale $\eta_{0}$ near some observer,

$$
p_{\alpha}:=\beta_{0} t_{\alpha}, \quad p_{\alpha} p^{\alpha} \approx r^{-2}
$$

where $\beta_{0}=\cosh ^{-1}\left(\eta_{0}\right) \ll 1$. They satisfy approximately canonical commutation relations

$$
\left\{p_{\alpha}, x^{\mu}\right\}=\beta_{0} \sinh (\eta) \delta_{\alpha}^{\mu} \approx \delta_{\alpha}^{\mu}
$$

The noncommutativity of the remaining generators is obtained from (2.27),

$$
\begin{aligned}
\left\{p^{0}, p^{j}\right\} & =-\frac{\beta_{0}}{R} p^{j} \\
\left\{p^{i}, p^{j}\right\} & =\frac{\beta_{0}^{2}}{R} \epsilon^{i j k} p^{k} \\
\left\{x^{0}, x^{j}\right\} & =r^{2} R \beta_{0}^{-1} p^{j} \\
\left\{x^{i}, x^{j}\right\} & =r^{2} R \varepsilon^{i j k} p^{k}
\end{aligned}
$$

at the reference point. Hence $\left\{p^{\mu}, p^{\nu}\right\}=O\left(\frac{1}{L_{N C}^{2}}\right)$ while $\left\{x^{\mu}, x^{\nu}\right\}=O\left(L_{N C}^{2}\right)$. 


\section{A.3 Proof of Lemma 5.1}

Consider first the following contraction

$$
\begin{aligned}
\Gamma_{\dot{\gamma} \mu}{ }^{\mu} & =\Gamma_{\dot{\gamma} \dot{\beta}}{ }^{\mu} E^{\dot{\beta}}{ }_{\mu}=-\left\{Z_{\dot{\gamma}}, E_{\dot{\beta}}{ }^{\mu}\right\} E^{\dot{\beta}}{ }_{\mu}=-\left\{Z_{\dot{\gamma}}, E_{\dot{\beta}}{ }^{\mu}\right\} E_{\dot{\beta}}{ }^{\nu} \gamma_{\nu \mu} \\
& =-\left\{Z_{\dot{\gamma}}, E_{\dot{\beta}}{ }^{\mu} E_{\dot{\beta}}{ }^{\nu}\right\} \gamma_{\nu \mu}+\left\{Z_{\dot{\gamma}}, E_{\dot{\beta}}{ }^{\nu}\right\} E_{\dot{\beta}}{ }^{\mu} \gamma_{\nu \mu} \\
& =-\left\{Z_{\dot{\gamma}}, \gamma^{\mu \nu}\right\} \gamma_{\nu \mu}+\left\{Z_{\dot{\gamma}}, E_{\dot{\beta}}{ }^{\nu}\right\} E_{\dot{\beta}}{ }^{\mu} \gamma_{\nu \mu} \\
& =-\left\{Z_{\dot{\gamma}}, \gamma^{\mu \nu}\right\} \gamma_{\nu \mu}+\left\{Z_{\dot{\gamma}}, E_{\dot{\beta}}{ }^{\nu}\right\} E^{{ }^{\beta}}{ }_{\nu} \\
& =-\left\{Z_{\dot{\gamma}}, \gamma^{\mu \nu}\right\} \gamma_{\nu \mu}-\Gamma_{\dot{\gamma} \mu}{ }^{\mu}
\end{aligned}
$$

This gives (5.34)

$$
\begin{aligned}
2 \Gamma_{\dot{\gamma} \mu}{ }^{\mu} & =-\left\{Z_{\dot{\gamma}}, \gamma^{\mu \nu}\right\} \gamma_{\nu \mu}=-E_{\dot{\gamma}}{ }^{\rho} \gamma_{\nu \mu} \partial_{\rho} \gamma^{\mu \nu}=-E_{\dot{\gamma}}{ }^{\rho} \partial_{\rho} \ln \left(\left|\gamma^{\mu \nu}\right|\right) \\
\Gamma_{\rho \mu}{ }^{\mu} & =\partial_{\rho} \ln \left(\sqrt{\left|\gamma_{\mu \nu}\right|}\right)
\end{aligned}
$$

which has the same form as for the Christoffel symbols for the Levi-Civita connection. Now consider the d'Alembertian $\square \phi$ :

$$
\begin{aligned}
\left\{Z_{\dot{\alpha}},\left\{Z^{\dot{\alpha}}, \phi\right\}\right\} & =E_{\dot{\alpha}}{ }^{\mu} \partial_{\mu}\left(E^{\dot{\alpha} \nu} \partial_{\nu} \phi\right) \\
& =\partial_{\mu}\left(E_{\dot{\alpha}}{ }^{\mu} E^{\dot{\alpha} \nu} \partial_{\nu} \phi\right)-\partial_{\mu}\left(E_{\dot{\alpha}}{ }^{\mu}\right) E^{\dot{\alpha} \nu} \partial_{\nu} \phi \\
& =\partial_{\mu}\left(\gamma^{\mu \nu} \partial_{\nu} \phi\right)+\Gamma_{\rho \dot{\alpha}}{ }^{\rho} E^{\dot{\alpha} \nu} \partial_{\nu} \phi \\
& =\partial_{\mu}\left(\gamma^{\mu \nu} \partial_{\nu} \phi\right)+\Gamma_{\mu}{ }^{\nu \mu} \partial_{\nu} \phi .
\end{aligned}
$$

Alternatively, we can proceed as

$$
\begin{aligned}
\left\{Z_{\dot{\alpha}},\left\{Z^{\dot{\alpha}}, \phi\right\}\right\} & =\gamma^{\mu \nu} \partial_{\mu} \partial_{\nu} \phi+E_{\dot{\alpha}}{ }^{\mu}\left(\partial_{\mu} E^{\dot{\alpha} \nu}\right) \partial_{\nu} \phi \\
& =\gamma^{\mu \nu} \partial_{\mu} \partial_{\nu} \phi-E_{\dot{\alpha}}{ }^{\mu} \Gamma_{\mu \rho}{ }^{\nu} E^{\dot{\alpha} \rho} \partial_{\nu} \phi \\
& =\gamma^{\mu \nu} \partial_{\mu} \partial_{\nu} \phi-\Gamma_{\mu \rho}{ }^{\nu} \gamma^{\rho \mu} \partial_{\nu} \phi \\
& =\gamma^{\mu \nu} \partial_{\mu} \partial_{\nu} \phi-\Gamma_{\mu}{ }^{\mu \nu} \partial_{\nu} \phi .
\end{aligned}
$$

In particular, this gives (5.33)

$$
\square y^{\sigma}=\partial_{\mu} \gamma^{\mu \sigma}+\Gamma_{\rho}{ }^{\sigma \rho}=-\Gamma_{\mu}{ }^{\mu \sigma} .
$$

As a check, the second line in (5.14) reproduces the standard identity in Riemannian geometry

$$
\begin{aligned}
\Gamma_{\mu}^{(\gamma)}{ }_{\mu}^{\mu \sigma} & =\Gamma_{\mu}{ }^{\sigma \mu}+\Gamma_{\mu}{ }^{\mu \sigma}-\Gamma_{\mu}^{\sigma \mu} \\
& =-\partial_{\mu} \gamma^{\mu \sigma}+\partial^{\sigma} \ln \left(\sqrt{\left|\gamma^{\mu \nu}\right|}\right)=-\frac{1}{\sqrt{\left|\gamma_{\mu \nu}\right|}} \partial_{\mu}\left(\sqrt{\left|\gamma_{\mu \nu}\right|} \gamma^{\mu \sigma}\right) .
\end{aligned}
$$

To show (5.32), we observe that the basic frame can be written using (2.42) as follows

$$
E_{\dot{\beta}}{ }^{\mu}=\left\{Z_{\dot{\beta}}, y^{\mu}\right\}=-\theta^{\mu \sigma} \partial_{\sigma} Z_{\dot{\beta}}
$$


Therefore

$$
\partial_{\mu}\left(\rho_{M} E_{\dot{\beta}}{ }^{\mu}\right)=-\partial_{\mu}\left(\rho_{M} \theta^{\mu \sigma} \partial_{\sigma} Z_{\dot{\beta}}\right)=-\partial_{\mu}\left(\rho_{M} \theta^{\mu \sigma}\right) \partial_{\sigma} Z_{\dot{\beta}}=0
$$

using the identity $\partial_{\mu}\left(\rho_{M} \theta^{\mu \sigma}\right)=0$ (2.52) which follows from the Jacobi identity. Together with $\partial_{\mu} E_{\dot{\beta}}{ }^{\mu}=-\Gamma_{\mu \dot{\beta}}{ }^{\mu}$ this gives

$$
\rho_{M} \Gamma_{\rho \dot{\beta}}^{\rho}=E_{\dot{\beta}}{ }^{\mu} \partial_{\mu} \rho_{M}
$$

which gives (5.32). We can use this to continue (A.13) as follows

$$
\begin{aligned}
\left\{Z_{\dot{\alpha}},\left\{Z^{\dot{\alpha}}, \phi\right\}\right\} & =\partial_{\mu}\left(\gamma^{\mu \nu} \partial_{\nu} \phi\right)+\Gamma_{\mu}^{\nu \mu} \partial_{\nu} \phi \\
& =\partial_{\mu}\left(\gamma^{\mu \nu} \partial_{\nu} \phi\right)+\rho_{M}^{-1} \gamma^{\mu \nu} \partial_{\mu} \rho_{M} \partial_{\nu} \phi \\
& =\rho_{M}^{-1} \partial_{\mu}\left(\rho_{M} \gamma^{\mu \nu} \partial_{\nu} \phi\right) \\
& =\frac{\rho^{2}}{\sqrt{\left|G_{\mu \nu}\right|}} \partial_{\mu}\left(\sqrt{\left|G_{\mu \nu}\right|} G^{\mu \rho} \partial_{\nu} \phi\right)
\end{aligned}
$$

using $\sqrt{\left|G_{\mu \nu}\right|} G^{\mu \rho}=\rho_{M} \gamma^{\mu \nu}$ and $\sqrt{\left|G_{\mu \nu}\right|}=\rho_{M} \rho^{2}$. Now (5.31) follows.

Finally, contacting (5.3) with $E^{\dot{\alpha} \nu}$ gives

$$
E^{\dot{\alpha} \nu} \partial_{\nu} E_{\dot{\alpha}}{ }^{\mu}=-\Gamma_{\nu \rho}{ }^{\mu} E_{\dot{\alpha}}{ }^{\rho} E^{\dot{\alpha} \nu}=-\Gamma_{\nu \rho}{ }^{\mu} \gamma^{\nu \rho}
$$

hence

$$
\begin{aligned}
\left\{Z^{\dot{\alpha}},\left\{Z_{\dot{\alpha}}, y^{\mu}\right\}\right\} & =-\Gamma_{\nu \rho}^{\mu} \gamma^{\nu \rho} \\
\square y^{\mu} & =\Gamma_{\nu \rho}{ }^{\mu} \gamma^{\nu \rho} .
\end{aligned}
$$

\section{A.4 Weitzenböck connection for the cosmic background}

For the cosmic background $\mathcal{M}$ defined by $Z_{\dot{\alpha}}=T_{\dot{\alpha}} \sim t_{\dot{\alpha}}$ (2.19), we have in Cartesian coordinates $x^{\mu}$

$$
\begin{aligned}
\bar{E}^{\dot{\alpha} \mu} & =\left\{t^{\dot{\alpha}}, x^{\mu}\right\}=\sinh (\eta) \eta^{\dot{\alpha} \mu}, & \bar{\gamma}^{\mu \nu} & =\sinh ^{2}(\eta) \eta^{\mu \nu} \\
\bar{G}^{\mu \nu} & =\sinh ^{-3}(\eta) \bar{\gamma}^{\mu \nu}=\sinh ^{-1}(\eta) \eta^{\mu \nu}, & \bar{\rho}^{2} & =\sinh ^{3}(\eta) .
\end{aligned}
$$

The Weitzenböck connection is obtained as

$$
\begin{aligned}
& \Gamma_{\nu \rho}{ }^{\mu}=-E^{\dot{\alpha}}{ }_{\rho} \partial_{\nu} E_{\dot{\alpha}}{ }^{\mu}=-\sinh ^{-1}(\eta) \delta_{\rho}^{\mu} \partial_{\nu} \sinh (\eta) \\
& \Gamma_{\rho \mu}^{\nu}=\frac{1}{R^{2} \rho^{2}} \tau^{\nu} \bar{G}_{\mu \rho}=\frac{1}{R^{2}} \tau^{\nu} \bar{\gamma}_{\mu \rho}, \quad \tau=x^{\mu} \partial_{\mu}
\end{aligned}
$$

noting that $R^{2} \sinh (\eta) \partial_{\mu} \sinh (\eta)=-\eta_{\mu \nu} \tau^{\nu}$. Here $\tau=x^{\mu} \partial_{\mu}$ is the $\operatorname{SO}(3,1)$-invariant cosmic time-like vector field (in Cartesian coordinates) which measures the cosmic scale,

$$
\bar{G}_{\rho \rho^{\prime}} \tau^{\rho} \tau^{\rho^{\prime}}=-R^{2} \cosh ^{2}(\eta) \sinh (\eta)=-a(t)^{2} \sim-R^{2} \bar{\rho}^{2}
$$

where $a(t)$ is the FLRW scale factor, cf. [12]. Hence the torsion is

$$
\bar{T}_{\rho \sigma}^{\mu}=\frac{1}{R^{2} \rho^{2}}\left(\delta_{\sigma}^{\mu} \tau_{\rho}-\delta_{\rho}^{\mu} \tau_{\sigma}\right)
$$


where $\tau_{\nu}=\bar{G}_{\nu \sigma} \tau^{\sigma}$. As a check, the torsion tensor can also be computed directly from (5.9). One can also verify

$$
\bar{T}_{\mu \sigma}^{\mu}=2 \rho^{-1} \partial_{\sigma} \bar{\rho}=-\frac{3}{R^{2} \rho^{2}} \tau_{\sigma}
$$

The contorsion is

$$
\bar{K}_{\mu \nu}^{\sigma}=\frac{1}{R^{2} \rho^{2}}\left(\bar{G}_{\mu \nu} \tau^{\sigma}-\delta_{\mu}^{\sigma} \tau_{\nu}\right)
$$

which is antisymmetric in $\nu \sigma$. We also need

$$
\begin{aligned}
\bar{T}_{\rho}{ }^{\mu}{ }_{\sigma} \bar{T}_{\nu \mu}{ }^{\rho} & =\frac{1}{R^{4} \rho^{4}}\left(-\tau_{\sigma} \tau_{\nu}+\bar{G}_{\sigma \nu} \tau^{\mu} \tau_{\mu}\right)=\bar{T}_{\rho}{ }_{\nu}{ }_{\nu} \bar{T}_{\sigma \mu}{ }^{\rho} \\
\bar{K}_{\mu \nu}{ }^{\rho}{ }_{\nu} \bar{K}_{\rho}{ }^{\mu}{ }_{\sigma} & =\frac{3}{R^{4} \rho^{4}} \tau_{\nu} \tau_{\sigma} \\
-\frac{1}{2}\left(\bar{T}_{\rho}{ }^{\mu}{ }_{\sigma} \bar{T}_{\nu \mu}{ }^{\rho}+\bar{T}_{\rho}{ }_{\nu}{ }_{\nu} \bar{T}_{\sigma \mu}{ }^{\rho}\right)-\bar{K}_{\mu}{ }^{\rho}{ }_{\nu} \bar{K}_{\rho}{ }^{\mu}{ }_{\sigma} & =-\frac{1}{R^{4} \rho^{4}}\left(2 \tau_{\sigma} \tau_{\nu}+\bar{G}_{\sigma \nu} \tau^{\mu} \tau_{\mu}\right) \\
2 \bar{\rho}^{-2} \partial_{\sigma}{ }_{\rho} \partial_{\nu} \bar{\rho} & =\frac{9}{2 R^{4} \rho^{4}} \tau_{\sigma} \tau_{\nu} \\
\rho \square_{G} \rho & =\frac{3}{2 R^{2}}\left(4+\frac{1}{2} \frac{\cosh ^{2}(\eta)}{\sinh ^{2}(\eta)}\right)
\end{aligned}
$$

Now we check the eom (5.47) for the background. Using

$$
\nabla_{\nu} \tau^{\rho}=\partial_{\nu} \tau^{\rho}+\Gamma_{\nu}{ }_{\mu}{ }_{\mu} \tau^{\mu}=\delta_{\nu}^{\rho}+\frac{1}{R^{2} \rho^{2}} \tau_{\nu} \tau^{\rho}
$$

one finds

$$
\nabla_{\nu} \bar{T}_{\rho \mu}^{\nu}=\frac{1}{R^{2} \rho^{2}}\left(\bar{G}_{\rho \mu}\left(3+\frac{1}{R^{2} \rho^{2}} \tau_{\nu} \tau^{\nu}\right)-\frac{1}{R^{2} \rho^{2}} \tau_{\mu} \tau_{\rho}\right)
$$

so that (5.47) is indeed satisfied,

$$
\nabla_{\nu} \bar{T}_{\rho \mu}^{\nu}+\bar{T}_{\nu}{ }_{\mu}^{\sigma} \bar{T}_{\sigma \rho}^{\nu}=\frac{3}{R^{2} \rho^{2}} \bar{G}_{\rho \mu}=m^{2} \bar{\gamma}_{\rho \mu}
$$

using $m^{2}=\frac{3}{R^{2}}$. We can also check (5.50). The Ricci tensor is obtained from (5.70) as

$$
\begin{aligned}
\mathcal{R}_{\mu \nu} & =-\frac{1}{2}\left(\bar{T}_{\rho}{ }_{\sigma}{ }_{\sigma} \bar{T}_{\nu \mu}{ }^{\rho}+\bar{T}_{\rho}{ }^{\mu}{ }_{\nu} \bar{T}_{\sigma \mu}{ }^{\rho}\right)-\bar{K}_{\mu}{ }^{\rho}{ }_{\nu} \bar{K}_{\rho}{ }^{\mu}{ }_{\sigma}+2 \bar{\rho}^{-2} \partial_{\nu} \bar{\rho} \partial_{\sigma} \bar{\rho}+\gamma_{\nu \sigma}\left(m^{2}-\frac{1}{2} T_{\nu}{ }_{\mu}{ }_{\mu} T_{\sigma \rho}{ }^{\nu} \gamma^{\mu \rho}\right) \\
& =\frac{5}{2} \frac{1}{\rho^{4} R^{4}} \tau_{\sigma} \tau_{\nu}+\frac{1}{2 \rho^{2} R^{2}} G_{\nu \sigma}\left(6-\operatorname{coth}^{2}(\eta)\right) \\
& \sim \frac{5}{2} \frac{1}{a(t)^{2}}\left(\frac{1}{a(t)^{2}} \tau_{\sigma} \tau_{\nu}+G_{\nu \sigma}\right) .
\end{aligned}
$$

The second line is indeed the exact result as can be checked with more standard methods, and the third line holds in the asymptotic regime for large $\eta$. This method of computing the Ricci tensor using the torsion is in fact quite efficient. Hence we obtain the effective 
vacuum energy-momentum tensor (5.73)

$$
\begin{aligned}
\overline{\mathbf{T}}_{\mu \nu} & =\mathcal{R}_{\mu \nu}-\frac{1}{2} G_{\mu \nu} \mathcal{R} \\
& =\frac{1}{\rho^{2} R^{2}}\left(\frac{5}{2} \frac{\tau_{\mu} \tau_{\nu}}{\rho^{2} R^{2}}+\frac{1}{2} G_{\mu \nu}\left(6-\operatorname{coth}^{2}(\eta)\right)-\frac{1}{2} G_{\mu \nu}\left(-\frac{5}{2} \frac{a(t)^{2}}{\rho^{2} R^{2}}+2\left(6-\operatorname{coth}^{2}(\eta)\right)\right)\right) \\
& \sim \frac{5}{2} \frac{1}{a(t)^{2}}\left(\frac{\tau_{\mu} \tau_{\nu}}{a(t)^{2}}-\frac{1}{2} G_{\mu \nu}\right) .
\end{aligned}
$$

In comoving coordinates, this has the form $\overline{\mathbf{T}}_{\mu \nu} \sim \frac{1}{a(t)^{2}} \operatorname{diag}(3,-1,-1,-1)$. Hence pressure is negative with $\omega=\frac{p}{\rho} \sim-\frac{1}{3}$, and the strong energy condition is (just) satisfied.

\section{A.5 Alternative derivation of the Ricci tensor in vaccum}

Here we give a direct derivation of the on-shell equation of the Ricci tensor in a more matrix-model-adapted approach, using only the matrix equations of motion rather than the derived equation (5.47) for the torsion. The result agrees perfectly with (5.69), which provides a consistency check of the present formalism. We start with

$$
\begin{aligned}
\left\{y^{\mu}, \square \phi\right\} & =-\left\{y^{\mu},\left\{Z^{\dot{\alpha}},\left\{Z_{\dot{\alpha}}, \phi\right\}\right\}=-\left\{\left\{y^{\mu}, Z^{\dot{\alpha}}\right\},\left\{Z_{\dot{\alpha}}, \phi\right\}\right\}-\left\{Z^{\dot{\alpha}},\left\{y^{\mu},\left\{Z_{\dot{\alpha}}, \phi\right\}\right\}\right.\right. \\
& =\left\{E^{\dot{\alpha} \mu},\left\{Z_{\dot{\alpha}}, \phi\right\}\right\}-\left\{Z^{\dot{\alpha}},\left\{\left\{y^{\mu}, Z_{\dot{\alpha}}\right\}, \phi\right\}\right\}-\left\{Z^{\dot{\alpha}},\left\{Z_{\dot{\alpha}},\left\{y^{\mu}, \phi\right\}\right\}\right. \\
& =\left\{E^{\dot{\alpha} \mu},\left\{Z_{\dot{\alpha}}, \phi\right\}\right\}+\left\{Z^{\dot{\alpha}},\left\{E_{\dot{\alpha}}{ }^{\mu}, \phi\right\}\right\}+\square\left(\left\{y^{\mu}, \phi\right\}\right. \\
& \left.=-\left\{\left\{E^{\dot{\alpha} \mu}, Z_{\dot{\alpha}}\right\}, \phi\right\}\right\}+2\left\{E_{\dot{\alpha}}{ }^{\mu},\left\{Z_{\dot{\alpha}}, \phi\right\}\right\}+\square\left(\left\{y^{\mu}, \phi\right\}\right.
\end{aligned}
$$

for any scalar field $\phi$. Now assume that $y^{\mu}$ are harmonic coordinates,

$$
\square_{G} y^{\mu}=0=\square y^{\mu}
$$

recalling (5.31). It follows that

$$
\left\{E_{\dot{\alpha}}{ }^{\mu}, Z^{\dot{\alpha}}\right\}=\left\{\left\{Z_{\dot{\alpha}}, y^{\mu}\right\}, Z^{\dot{\alpha}}\right\}=\square y^{\mu}=0
$$

and we obtain

$$
\square\left\{y^{\mu}, \phi\right\}=\left\{y^{\mu}, \square \phi\right\}-2\left\{E^{\dot{\alpha} \mu},\left\{Z_{\dot{\alpha}}, \phi\right\}\right\} .
$$

Now we use the equation of motion $(2.3)$ for $Z^{\dot{\alpha}}$,

$$
\square Z^{\dot{\alpha}}=m^{2} Z^{\dot{\alpha}}
$$

Then (A.38) gives for $\phi=Z^{\dot{\beta}}$

$$
\begin{aligned}
\square\left\{y^{\mu}, Z^{\dot{\beta}}\right\} & =\left\{y^{\mu}, \square Z^{\dot{\beta}}\right\}-2\left\{E^{\dot{\alpha} \mu},\left\{Z_{\dot{\alpha}}, Z^{\dot{\beta}}\right\}\right\} \\
& =m^{2}\left\{y^{\mu}, Z^{\dot{\beta}}\right\}+2\left\{E_{\dot{\alpha}}{ }^{\mu}, \hat{\Theta}^{\dot{\alpha} \dot{\beta}}\right\} \\
\square E_{\dot{\beta}}{ }^{\mu} & =m^{2} E_{\dot{\beta}}{ }^{\mu}-2\left\{\hat{\Theta}^{\dot{\beta} \dot{\alpha}}, E_{\dot{\alpha}}{ }^{\mu}\right\} .
\end{aligned}
$$

For the rescaled frame $\mathcal{E}_{\dot{\alpha}}^{\mu}=\rho^{-1} E_{\dot{\alpha}}^{\mu}(4.12)$, this gives

$$
\square \mathcal{E}_{\dot{\alpha}}{ }^{\mu}=\left(m^{2}-\rho^{-1} \square \rho\right) \mathcal{E}_{\dot{\alpha}}{ }^{\mu}-2\left\{\hat{\Theta}^{\dot{\alpha} \dot{\beta}}, \mathcal{E}_{\dot{\beta}}{ }^{\mu}\right\}-2 \rho^{-1}\left\{\hat{\Theta}^{\dot{\alpha} \dot{\beta}}, \rho\right\} \mathcal{E}_{\dot{\beta}}{ }^{\mu}+2\left\{Z^{\dot{\gamma}}, \mathcal{E}_{\dot{\alpha}}{ }^{\mu}\right\} \rho^{-1}\left\{Z_{\dot{\gamma}}, \rho\right\}
$$


Equation of motion for the metric. Now we use (A.41) to compute $\square G^{\mu \nu}$. For the last term, we observe

$$
\left(\left\{Z^{\dot{\gamma}}, \mathcal{E}_{\dot{\alpha}}^{\mu}\right\}\left\{Z_{\dot{\gamma}}, \rho\right\} \mathcal{E}_{\dot{\beta}}^{\nu}+\left\{Z^{\dot{\gamma}}, \mathcal{E}_{\dot{\alpha}}^{\nu}\right\}\left\{Z_{\dot{\gamma}}, \rho\right\} \mathcal{E}_{\dot{\beta}}^{\mu}\right) \eta^{\dot{\alpha} \dot{\beta}}=\left\{Z^{\dot{\gamma}}, G^{\mu \nu}\right\}\left\{Z_{\dot{\gamma}}, \rho\right\}
$$

and similarly

$$
\left\{\hat{\Theta}^{\dot{\alpha} \dot{\beta}}, \rho\right\} \mathcal{E}_{\dot{\beta}}^{\mu} \mathcal{E}_{\dot{\alpha}}{ }^{\nu}+\left\{\hat{\Theta}^{\dot{\beta} \dot{\alpha}}, \rho\right\} \mathcal{E}_{\dot{\alpha}}^{\nu} \mathcal{E}_{\dot{\beta}}{ }^{\mu}=0
$$

Thus we obtain

$$
\begin{aligned}
\square G^{\mu \nu}= & 2\left(m^{2}-\rho^{-1} \square \rho\right) G^{\mu \nu}+2\left\{Z^{\dot{\gamma}}, G^{\mu \nu}\right\} \rho^{-1}\left\{Z_{\dot{\gamma}}, \rho\right\} \\
& -2 \mathcal{E}_{\dot{\alpha}}{ }^{\mu}\left\{\hat{\Theta}^{\dot{\alpha} \dot{\beta}}, \mathcal{E}_{\dot{\beta}}{ }^{\nu}\right\}-2\left\{\hat{\Theta}^{\dot{\beta} \dot{\alpha}}, \mathcal{E}_{\dot{\alpha}}{ }^{\mu}\right\} \mathcal{E}_{\dot{\beta}}{ }^{\nu}-2\left\{Z_{\dot{\gamma}}, \mathcal{E}_{\dot{\alpha}}{ }^{\mu}\right\}\left\{Z^{\dot{\gamma}}, \mathcal{E}^{\dot{\alpha} \nu}\right\} \\
= & 2\left(m^{2}-\rho^{-1} \square \rho\right) G^{\mu \nu}+2\left\{Z_{\dot{\beta}}, G^{\mu \nu}\right\} \rho^{-1}\left\{Z_{\dot{\beta}}, \rho\right\} \\
& +2 T^{\mu \sigma \rho} \tilde{\Gamma}_{\rho \sigma}{ }^{\nu}+2 T^{\nu \sigma \rho} \tilde{\Gamma}_{\rho \sigma}{ }^{\mu}-2 \tilde{\Gamma}_{\rho \sigma}{ }^{\mu} \tilde{\Gamma}^{\rho \sigma \nu}
\end{aligned}
$$

using

$$
\begin{aligned}
\left\{Z_{\dot{\gamma}}, \mathcal{E}_{\dot{\alpha}}{ }^{\mu}\right\}\left\{Z^{\dot{\gamma}}, \mathcal{E}^{\dot{\alpha} \nu}\right\} & \sim \gamma^{\rho \rho^{\prime}} \partial_{\rho} \mathcal{E}_{\dot{\alpha}}{ }^{\mu} \partial_{\rho^{\prime}} \mathcal{E}^{\dot{\alpha} \nu}=\gamma^{\rho \rho^{\prime}} \tilde{\Gamma}_{\rho \dot{\alpha}}{ }^{\mu} \tilde{\Gamma}_{\rho^{\prime}}{ }^{\dot{\alpha} \nu} \\
& =\gamma^{\rho \rho^{\prime}} G^{\sigma \sigma^{\prime}} \tilde{\Gamma}_{\rho \sigma}{ }^{\mu} \tilde{\Gamma}_{\rho^{\prime} \sigma^{\prime}}{ }^{\nu} \\
\mathcal{E}_{\dot{\alpha}}{ }^{\mu}\left\{\hat{\Theta}^{\dot{\alpha} \dot{\beta}}, \mathcal{E}_{\dot{\beta}}{ }^{\nu}\right\} & \sim \mathcal{E}^{\dot{\alpha} \mu} T_{\dot{\alpha} \dot{\beta}}{ }^{\rho} \partial_{\rho} \mathcal{E}^{\dot{\beta} \nu}=-\gamma^{\mu \mu^{\prime}} G^{\sigma \sigma^{\prime}} T_{\mu^{\prime} \sigma}{ }^{\rho} \tilde{\Gamma}_{\rho \sigma^{\prime}}{ }^{\nu}
\end{aligned}
$$

in the asymptotic regime, where $\tilde{\Gamma}_{\rho \sigma}^{\nu}$ is the Weitzenböck connection for the rescaled frame $\mathcal{E}$ and $T_{\dot{\alpha} \dot{\beta}}{ }^{\rho}$ is the torsion of basic frame $E$. Note that coordinate indices are raised and lowered with $G^{\mu \nu}$ here. Now consider harmonic normal coordinates $y^{\mu}$ (5.29) w.r.t. $G^{\rho \sigma}$ at the point $p$. Then $\left\{Z_{\dot{\beta}}, G^{\mu \nu}\right\}$ vanishes at $p$. Furthermore, the Weitzenböck connection $\tilde{\Gamma}_{\rho \sigma}{ }^{\mu}$ can then be expressed in terms of the (con)torsion via $\tilde{\Gamma}_{\rho \sigma}{ }^{\mu}=\mathcal{K}_{\rho \sigma}{ }^{\mu}$ (5.28). Thus

$$
G_{\mu \mu^{\prime}} G_{\nu \nu^{\prime}} \frac{1}{2} \square G^{\mu^{\prime} \nu^{\prime}}=\left(m^{2}-\rho^{-1} \square \rho\right) G_{\mu \nu}+\rho^{2}\left(T_{\mu \sigma}{ }^{\rho} \mathcal{K}_{\rho \rho}{ }^{\sigma}{ }_{\nu}+T_{\nu \sigma}{ }^{\rho} \mathcal{K}_{\rho}{ }^{\sigma}{ }_{\mu}-\mathcal{K}_{\rho}{ }^{\sigma}{ }_{\mu} \mathcal{K}^{\rho}{ }_{\sigma \nu}\right) .
$$

Now in harmonic NC at $p$, the Ricci tensor at $p$ is obtained as [40]

$$
\left.\mathcal{R}_{\mu \nu}[G]\right|_{p}=\frac{1}{2} \square_{G} G_{\mu \nu}=-G_{\mu \mu^{\prime}} G_{\nu \nu^{\prime}} \square_{G} G^{\mu^{\prime} \nu^{\prime}} .
$$

Together with $\square=\rho^{2} \square_{G}$ (5.31), we obtain the tensorial equation

$$
\mathcal{R}_{\mu \nu}=-T_{\mu \sigma}{ }^{\rho} \mathcal{K}_{\rho \nu}{ }^{\sigma}{ }_{\nu}-T_{\nu \sigma}{ }^{\rho} \mathcal{K}_{\rho}{ }^{\sigma}{ }_{\mu}+\mathcal{K}_{\rho}{ }^{\sigma}{ }_{\mu} \mathcal{K}^{\rho}{ }_{\sigma \nu}+\rho^{-2}\left(-m^{2}+\rho \square_{G} \rho\right) G_{\mu \nu}
$$

in the asymptotic regime. To see that this coincides with (5.69), we need to compare the contractions of the (con)torsion terms. One can easily see using (5.19) and (5.35) that

$$
\begin{aligned}
T_{\mu \sigma}{ }^{\rho} \mathcal{K}_{\rho}{ }^{\sigma}{ }_{\nu}+T_{\nu \sigma}{ }^{\rho} \mathcal{K}_{\rho \mu}{ }^{\sigma}-\mathcal{K}_{\rho \mu}{ }^{\sigma} \mathcal{K}^{\rho}{ }_{\sigma \nu}= & T_{\mu \sigma}{ }^{\rho} K_{\rho}{ }^{\sigma}{ }_{\nu}+T_{\nu \sigma}{ }^{\rho} K_{\rho \mu}{ }^{\sigma}{ }^{2}-K^{\rho}{ }_{\sigma \mu} K_{\rho}{ }^{\sigma}{ }_{\nu} \\
& -2 \rho^{-2} \partial_{\nu} \rho \partial_{\mu} \rho-G_{\mu \nu} \rho^{-2} \partial^{\sigma} \rho \partial_{\sigma} \rho
\end{aligned}
$$

using

$$
K_{\nu}^{\sigma}{ }_{\mu}+K_{\mu \nu}^{\sigma}=T_{\mu \nu}^{\sigma}+T_{\nu}^{\sigma}{ }_{\mu}
$$


Therefore we obtain

$\mathcal{R}_{\mu \nu}=-T_{\mu \sigma}{ }^{\rho} K_{\rho}{ }^{\sigma}{ }_{\nu}-T_{\nu \sigma}{ }^{\rho} K_{\rho}{ }^{\sigma}{ }_{\mu}+K_{\rho}{ }^{\sigma}{ }_{\mu} K^{\rho}{ }_{\sigma \nu}+2 \rho^{-2} \partial_{\nu} \rho \partial_{\mu} \rho+\rho^{-2}\left(-m^{2}+\rho \square_{G} \rho+\partial^{\sigma} \rho \partial_{\sigma} \rho\right) G_{\mu \nu}$.

Expressing the tensorial part in terms of torsion only, one finds using (A.54)

$$
\begin{aligned}
& T_{\mu \sigma}{ }^{\rho} K_{\rho \nu}{ }^{\sigma}+T_{\nu \sigma}{ }^{\rho} K_{\rho \mu}^{\sigma}-K_{\sigma \mu}^{\rho} K_{\rho \nu}{ }^{\sigma} \\
& =\frac{1}{2} T_{\mu}{ }^{\sigma \rho}\left(T_{\nu \rho \sigma}+T_{\nu \sigma \rho}\right)+\frac{1}{2} T_{\mu}{ }^{\sigma \rho} T_{\rho \sigma \nu}+\frac{1}{2} T_{\nu}{ }^{\sigma \rho} T_{\rho \sigma \mu}+\frac{1}{4} T_{\mu}^{\sigma \rho}{ }_{\mu \sigma \nu}
\end{aligned}
$$

while for the other form (5.69) we need

$$
\begin{aligned}
& -\frac{1}{2} T_{\mu \sigma}{ }^{\rho} T_{\rho}{ }^{\sigma}{ }_{\nu}-\frac{1}{2} T_{\nu \sigma}{ }^{\rho} T_{\rho}{ }^{\sigma}{ }_{\mu}-K_{\sigma}{ }^{\rho}{ }_{\mu} K_{\rho}{ }^{\sigma}{ }_{\nu} \\
& \quad=-\frac{1}{2} T_{\mu}{ }^{\sigma \rho} T_{\rho \sigma \nu}-\frac{1}{2} T_{\nu}{ }^{\sigma \rho} T_{\rho \sigma \mu}+\frac{1}{4}\left(-2 T_{\mu}{ }^{\sigma \rho}\left(T_{\nu \rho \sigma}+T_{\nu \sigma \rho}\right)-T_{\mu}^{\sigma \rho} T_{\rho \sigma \nu}\right)
\end{aligned}
$$

Hence the two expressions for the Ricci tensor are indeed identical.

\section{A.6 Contractions of the (con)torsion}

We need the following rank 2 contractions with the contorsion

$$
\begin{aligned}
T^{\mu \sigma \rho} K_{\rho \sigma \nu} & =\frac{1}{2}\left(T^{\mu \sigma \rho}\left(T_{\nu \rho \sigma}+T_{\nu \sigma \rho}\right)+T^{\mu \sigma \rho} T_{\rho \sigma \nu}\right) \\
-K^{\sigma \rho \mu} K_{\rho \sigma \nu} & =\frac{1}{4}\left(-2 T^{\mu \sigma \rho}\left(T_{\nu \rho \sigma}+T_{\nu \sigma \rho}\right)+T^{\rho \sigma \mu} T_{\rho \sigma \nu}\right) \\
-K^{\rho \sigma \mu} K_{\rho \sigma \nu} & =\frac{1}{4}\left(-2 T^{\mu \rho \sigma}\left(T_{\nu \rho \sigma}+T_{\nu \sigma \rho}\right)+T^{\sigma \rho \mu} T_{\rho \sigma \nu}\right)
\end{aligned}
$$

Note that there are 3 independent rank 2 contractions. The contraction of the second expression gives

$$
-K_{\sigma}{ }^{\rho \mu} K_{\rho}{ }^{\sigma} \mu=-\frac{1}{4} T^{\mu \sigma \rho}\left(2 T_{\mu \rho \sigma}+T_{\mu \sigma \rho}\right)
$$

Therefore

$$
-T_{\mu}{ }^{\rho \sigma} T_{\rho \sigma}{ }^{\mu}-K_{\sigma}{ }^{\rho \mu} K_{\rho \mu}{ }^{\sigma}=-\frac{1}{2} T^{\sigma \mu \rho} T_{\mu \rho \sigma}-\frac{1}{4} T^{\mu \sigma \rho} T_{\mu \sigma \rho}
$$

Open Access. This article is distributed under the terms of the Creative Commons Attribution License (CC-BY 4.0), which permits any use, distribution and reproduction in any medium, provided the original author(s) and source are credited.

\section{References}

[1] M.H. Goroff and A. Sagnotti, The ultraviolet behavior of Einstein gravity, Nucl. Phys. B 266 (1986) 709 [INSPIRE].

[2] S. Minwalla, M. Van Raamsdonk and N. Seiberg, Noncommutative perturbative dynamics, JHEP 02 (2000) 020 [hep-th/9912072] [INSPIRE].

[3] A. Matusis, L. Susskind and N. Toumbas, The IR/UV connection in the noncommutative gauge theories, JHEP 12 (2000) 002 [hep-th/0002075] [INSPIRE]. 
[4] H.C. Steinacker, String states, loops and effective actions in noncommutative field theory and matrix models, Nucl. Phys. B 910 (2016) 346 [arXiv: 1606.00646] [InSPIRE].

[5] N. Ishibashi, H. Kawai, Y. Kitazawa and A. Tsuchiya, A large $N$ reduced model as superstring, Nucl. Phys. B 498 (1997) 467 [hep-th/9612115] [INSPIRE].

[6] V.O. Rivelles, Noncommutative field theories and gravity, Phys. Lett. B 558 (2003) 191 [hep-th/0212262] [INSPIRE].

[7] H.S. Yang, Emergent gravity from noncommutative spacetime, Int. J. Mod. Phys. A 24 (2009) 4473 [hep-th/0611174] [INSPIRE].

[8] H. Steinacker, Emergent geometry and gravity from matrix models: an introduction, Class. Quant. Grav. 27 (2010) 133001 [arXiv:1003.4134] [INSPIRE].

[9] M. Sperling and H.C. Steinacker, Covariant cosmological quantum space-time, higher-spin and gravity in the IKKT matrix model, JHEP 07 (2019) 010 [arXiv:1901.03522] [INSPIRE].

[10] H.C. Steinacker, Higher-spin kinematics \& no ghosts on quantum space-time in Yang-Mills matrix models, arXiv:1910.00839 [INSPIRE].

[11] P. de Medeiros and S. Ramgoolam, Non-associative gauge theory and higher spin interactions, JHEP 03 (2005) 072 [hep-th/0412027] [INSPIRE].

[12] H.C. Steinacker, Scalar modes and the linearized Schwarzschild solution on a quantized FLRW space-time in Yang-Mills matrix models, Class. Quant. Grav. 36 (2019) 205005 [arXiv: 1905.07255] [INSPIRE].

[13] M.A. Vasiliev, Consistent equation for interacting gauge fields of all spins in (3+1)-dimensions, Phys. Lett. B 243 (1990) 378 [INSPIRE].

[14] V.E. Didenko and E.D. Skvortsov, Elements of Vasiliev theory, arXiv:1401.2975 [INSPIRE].

[15] D. Ponomarev and E.D. Skvortsov, Light-front higher-spin theories in flat space, J. Phys. A 50 (2017) 095401 [arXiv: 1609.04655] [INSPIRE].

[16] E.D. Skvortsov, T. Tran and M. Tsulaia, Quantum chiral higher spin gravity, Phys. Rev. Lett. 121 (2018) 031601 [arXiv: 1805.00048] [INSPIRE].

[17] A.A. Tseytlin, On limits of superstring in $A d S_{5} \times S^{5}$, Theor. Math. Phys. 133 (2002) 1376 [hep-th/0201112] [INSPIRE].

[18] A.Y. Segal, Conformal higher spin theory, Nucl. Phys. B 664 (2003) 59 [hep-th/0207212] [INSPIRE].

[19] C.N. Pope and P.K. Townsend, Conformal higher spin in $(2+1)$-dimensions, Phys. Lett. B 225 (1989) 245 [INSPIRE].

[20] E.S. Fradkin and V. Ya. Linetsky, A superconformal theory of massless higher spin fields in $D=(2+1)$, Mod. Phys. Lett. A 4 (1989) 731 [INSPIRE].

[21] M. Grigoriev, I. Lovrekovic and E. Skvortsov, New conformal higher spin gravities in $3 d$, JHEP 01 (2020) 059 [arXiv:1909.13305] [INSPIRE].

[22] M. Hanada, H. Kawai and Y. Kimura, Describing curved spaces by matrices, Prog. Theor. Phys. 114 (2006) 1295 [hep-th/0508211] [INSPIRE].

[23] S.W. MacDowell and F. Mansouri, Unified geometric theory of gravity and supergravity, Phys. Rev. Lett. 38 (1977) 739 [Erratum ibid. 38 (1977) 1376] [INSPIRE]. 
[24] E. Langmann and R.J. Szabo, Teleparallel gravity and dimensional reductions of noncommutative gauge theory, Phys. Rev. D 64 (2001) 104019 [hep-th/0105094] [INSPIRE].

[25] P.-M. Ho, Generalized Yang-Mills theory and gravity, Phys. Rev. D 93 (2016) 044062 [arXiv:1501.05378] [INSPIRE].

[26] L. Bonora, M. Cvitan, P. Dominis Prester, S. Giaccari and T. Stemberga, HS in flat spacetime. YM-like models, arXiv:1812.05030 [INSPIRE].

[27] H.C. Steinacker, Emergent gravity on covariant quantum spaces in the IKKT model, JHEP 12 (2016) 156 [arXiv: 1606.00769] [INSPIRE].

[28] S.-W. Kim, J. Nishimura and A. Tsuchiya, Expanding $(3+1)$-dimensional universe from a Lorentzian matrix model for superstring theory in $(9+1)$-dimensions, Phys. Rev. Lett. 108 (2012) 011601 [arXiv: 1108.1540] [INSPIRE].

[29] J. Nishimura and A. Tsuchiya, Complex Langevin analysis of the space-time structure in the Lorentzian type IIB matrix model, JHEP 06 (2019) 077 [arXiv: 1904.05919] [INSPIRE].

[30] H.C. Steinacker, On the quantum structure of space-time, gravity and higher spin in matrix models, arXiv: 1911.03162 [INSPIRE].

[31] M. Sperling and H.C. Steinacker, The fuzzy 4-hyperboloid $H_{n}^{4}$ and higher-spin in Yang-Mills matrix models, Nucl. Phys. B 941 (2019) 680 [arXiv:1806.05907] [INSPIRE].

[32] H.C. Steinacker, Quantized open FRW cosmology from Yang-Mills matrix models, Phys. Lett. B 782 (2018) 176 [arXiv:1710.11495] [INSPIRE].

[33] R. Aldrovandi and J.G. Pereira, Teleparallel gravity, Fund. Theor. Phys. 173, Springer, The Netherlands (2013).

[34] M. Dimitrijević Ćirić, B. Nikolić and V. Radovanović, Noncommutative $\mathrm{SO}(2,3)_{\star}$ gravity: noncommutativity as a source of curvature and torsion, Phys. Rev. D 96 (2017) 064029 [arXiv: 1612.00768] [INSPIRE].

[35] A. Chatzistavrakidis, H. Steinacker and G. Zoupanos, Intersecting branes and a Standard Model realization in matrix models, JHEP 09 (2011) 115 [arXiv:1107.0265] [INSPIRE].

[36] P. Aschieri, T. Grammatikopoulos, H. Steinacker and G. Zoupanos, Dynamical generation of fuzzy extra dimensions, dimensional reduction and symmetry breaking, JHEP 09 (2006) 026 [hep-th/0606021] [INSPIRE].

[37] M. Sperling and H.C. Steinacker, Intersecting branes, Higgs sector and chirality from $N=4$ SYM with soft SUSY breaking, JHEP 04 (2018) 116 [arXiv: 1803.07323] [INSPIRE].

[38] H. Aoki, J. Nishimura and A. Tsuchiya, Realizing three generations of the Standard Model fermions in the type IIB matrix model, JHEP 05 (2014) 131 [arXiv: 1401.7848] [INSPIRE].

[39] K. Hatakeyama, A. Matsumoto, J. Nishimura, A. Tsuchiya and A. Yosprakob, The emergence of expanding space-time and intersecting D-branes from classical solutions in the Lorentzian type IIB matrix model, arXiv:1911.08132 [INSPIRE].

[40] D.M. Deturck and J.L. Kazdan, Some regularity theorems in Riemannian geometry, Ann. Sci. École Norm. Sup. 14 (1981) 249. 